\title{
Trigonometric Lie algebras, affine Lie algebras, and vertex algebras
}

\author{
Haisheng Lin1, Shaobin Tan"2, and Qing Wang 4 \\ ${ }^{a}$ Department of Mathematical Sciences \\ Rutgers University, Camden, NJ 08102, USA \\ ${ }^{b}$ School of Mathematical Sciences, Xiamen University, Xiamen 361005, China
}

\begin{abstract}
In this paper, we explore natural connections among trigonometric Lie algebras, (general) affine Lie algebras, and vertex algebras. Among the main results, we obtain a realization of trigonometric Lie algebras as what were called the covariant algebras of the affine Lie algebra $\widehat{\mathcal{A}}$ of Lie algebra $\mathcal{A}=\mathfrak{g l}_{\infty} \oplus \mathfrak{g l}_{\infty}$ with respect to certain automorphism groups. We then prove that restricted modules of level $\ell$ for trigonometric Lie algebras naturally correspond to equivariant quasi modules for the affine vertex algebras $V_{\widehat{\mathcal{A}}}(\ell, 0)$ (or $V_{\widehat{\mathcal{A}}}(2 \ell, 0)$ ). Furthermore, we determine irreducible modules and equivariant quasi modules for simple vertex algebra $L_{\widehat{\mathcal{A}}}(\ell, 0)$ with $\ell$ a positive integer. In particular, we prove that every quasi-finite unitary highest weight (irreducible) module of level $\ell$ for type $A$ trigonometric Lie algebra gives rise to an irreducible equivariant quasi $L_{\widehat{\mathcal{A}}}(\ell, 0)$-module.
\end{abstract}

\section{Introduction}

Trigonometric Lie algebras, of types $A, B, C$, and $D$, are a family of infinite-dimensional Lie algebras (see [FFZ], [F], G-KL1], G-KL2]). For example, the rank 2 trigonometric (sine) Lie algebra $\widehat{A}_{\hbar}$ (of type $A$ ) with a real parameter $\hbar$ is the Lie algebra with generators $A_{\alpha, m}$ for $(\alpha, m) \in \mathbb{Z}^{2}$ and $\mathbf{c}$, a central element, subject to relations

$$
\left[A_{\alpha, m}, A_{\beta, n}\right]=2 i \sin \hbar(m \beta-n \alpha) A_{\alpha+\beta, m+n}+m \delta_{\alpha+\beta, 0} \delta_{m+n, 0} \mathbf{c}
$$

for $\alpha, \beta, m, n \in \mathbb{Z}$. In literature, the rank 2 sine Lie algebra is also known as the quantum 2-torus Lie algebra. It was known (see [H], [F], G-KL1, G-KL2], KR]) that the sine Lie algebra has a canonical relation with $\overline{\mathfrak{g l}_{\infty}}$ (the formal completion with a central extension). Especially, quasi-finite unitary highest weight (irreducible) modules were classified in [KR] and an explicit vertex operator realization was obtained in [F] and [G-KL2]. In this current paper, we study trigonometric Lie algebras with a different perspective, to explore potential natural connections with vertex algebras.

It has been known that (untwisted) affine Lie algebras have a natural association with vertex algebras and modules (cf. [FZ], [Li1]), whereas twisted affine Lie algebras are naturally associated with twisted modules for vertex algebras (see [FLM], [Li2]). Let $\mathfrak{g}$ be any

\footnotetext{
${ }^{1}$ Partially supported by China NSF grants (Nos.11471268, 11571391,11671247)

${ }^{2}$ Partially supported by China NSF grants (Nos.11471268, 11531004)

${ }^{3}$ Partially supported by China NSF grants (Nos.11531004, 11622107), Natural Science Foundation of Fujian Province (No. 2016J06002)
} 
(possibly infinite-dimensional) Lie algebra, equipped with a non-degenerate symmetric invariant bilinear form $\langle\cdot, \cdot\rangle$. Then we have an affine Lie algebra $\widehat{\mathfrak{g}}=\mathfrak{g} \otimes \mathbb{C}\left[t, t^{-1}\right] \oplus \mathbb{C} \mathbf{k}$. For any complex number $\ell$, we have a vertex algebra $V_{\widehat{\mathfrak{g}}}(\ell, 0)$ and its simple quotient vertex algebra $L_{\widehat{\mathfrak{g}}}(\ell, 0)$. It is known that the category of $V_{\widehat{\mathfrak{g}}}(\ell, 0)$-modules is canonically isomorphic to the category of restricted $\widehat{\mathfrak{g}}$-modules of level $\ell$. An important problem is to classify irreducible $L_{\widehat{\mathfrak{g}}}(\ell, 0)$-modules. Every irreducible $L_{\widehat{\mathfrak{g}}}(\ell, 0)$-module is necessarily an irreducible restricted $\widehat{\mathfrak{g}}$-module of level $\ell$, but that is not all in general.

Suppose that $\mathfrak{g}$ is a finite-dimensional simple Lie algebra with $\langle\cdot, \cdot\rangle$ the killing form normalized such that the squared length of long roots equals 2 . (In this case, the affine Lie algebra $\widehat{\mathfrak{g}}$ is an affine Kac-Moody algebra.) Let $\ell$ be a positive integer. It was known (see [FZ], [DL], Li1]) that irreducible $L_{\widehat{\mathfrak{g}}}(\ell, 0)$-modules are exactly the integrable highest weight $\widehat{\mathfrak{g}}$-modules of level $\ell$, which are also the unitary highest weight $\widehat{\mathfrak{g}}$-modules of level $\ell$.

Note that the particular way to associate vertex algebras and their modules to affine Lie algebras works for a wide variety of infinite-dimensional Lie algebras. However, it no longer works for trigonometric Lie algebras, including quantum 2-torus Lie algebra. With this as the main driving force, a theory of what were called quasi modules for vertex algebras was developed in [Li3]. The notion of quasi module generalizes that of module for a vertex algebra in a certain natural way. Indeed, by using this theory a much wider variety of Lie algebras can be associated with vertex algebras. In order to study quasi modules more efficiently, the notion of vertex algebra was enhanced to a notion of $\Gamma$ vertex algebra. Later, a notion of equivariant quasi module was introduced for a vertex $G$-algebra in [Li4]. It was proved in [Li5] that for a vertex operator algebra $V$ with a finite order automorphism $\sigma$, the category of $\sigma$-twisted $V$-modules is canonically isomorphic to the category of equivariant quasi $V$-modules with $G=\langle\sigma\rangle$. In view of this, the notion of quasi module can be viewed as a generalization of the notion of twisted module.

In this current paper, we first prove that trigonometric Lie algebras are isomorphic to what were called the covariant algebras of the affine Lie algebra $\widehat{\mathcal{A}}$ with respect to certain infinite order automorphism groups, where $\mathcal{A}$ is a Lie algebra isomorphic to $\mathfrak{g l}_{\infty} \oplus \mathfrak{g l}_{\infty}$. Then we give an isomorphism between the category of restricted modules for trigonometric Lie algebras of level $\ell$ and the category of equivariant quasi modules for $V_{\widehat{\mathcal{A}}}(\ell, 0)$ for type $A$ and for $V_{\widehat{A}}(2 \ell, 0)$ for types $B$ and $D$. Furthermore, we determine $L_{\widehat{A}}(\ell, 0)$-modules and equivariant quasi $L_{\widehat{\mathcal{A}}}(\ell, 0)$-modules with $\ell$ a positive integer. In the establishment of the isomorphism between trigonometric Lie algebras and the covariant Lie algebras of affine Lie algebras, the theory of equivariant quasi modules for vertex $\Gamma$-algebras plays a vital role.

Now, we mention some key ingredients in this paper. Recall that associated to any Lie algebra $\mathfrak{g}$ with a non-degenerate symmetric invariant bilinear form $\langle\cdot, \cdot\rangle$, we have an affine Lie algebra $\widehat{\mathfrak{g}}=\mathfrak{g} \otimes \mathbb{C}\left[t, t^{-1}\right] \oplus \mathbb{C} \mathbf{k}$. Let $G$ be an automorphism group of $\mathfrak{g}$, which preserves the bilinear form $\langle\cdot, \cdot\rangle$, satisfying the condition that for $a, b \in \mathfrak{g}$,

$$
[g a, b]=0 \text { and }\langle g a, b\rangle=0
$$

for all but finitely many $g \in G$. On the other hand, let $\chi$ be a linear character of $G$. Let 
$G$ act on $\widehat{\mathfrak{g}}$ by

$$
g\left(a \otimes t^{m}\right)=\chi(g)^{m}\left(g a \otimes t^{m}\right) \quad \text { for } g \in G, a \in \mathfrak{g}, m \in \mathbb{Z} .
$$

The covariant algebra of affine Lie algebra $\widehat{\mathfrak{g}}$ with respect to $(G, \chi)$ is defined (see [G-KK], [Li4]) to be the Lie algebra with $\widehat{\mathfrak{g}}$ as the generating space and with defining relations

$$
g\left(a \otimes t^{m}\right)=a \otimes t^{m} \quad \text { for } g \in G, a \in \mathfrak{g}, m \in \mathbb{Z}
$$

and

$$
\left[a \otimes t^{m}+\mu \mathbf{k}, b \otimes t^{n}+\nu \mathbf{k}\right]=\sum_{g \in G} \chi(g)^{m}\left([g a, b] \otimes t^{m+n}+m\langle g a, b\rangle \delta_{m+n, 0} \mathbf{k}\right)
$$

for $a, b \in \mathfrak{g}, m, n \in \mathbb{Z}, \mu, \nu \in \mathbb{C}$. In case that $G$ is finite, the above covariant Lie algebra is isomorphic to the subalgebra of $G$-fixed points in $\widehat{\mathfrak{g}}$. In view of this, covariant Lie algebras of affine Lie algebras can be viewed as generalizations of twisted affine Lie algebras.

We here need a particular Lie algebra $\mathfrak{g}$. Let $\mathfrak{g l}_{\infty}$ denote the associative algebra of doubly infinite complex matrices with finitely many nonzero entries. Fix a symmetric invariant bilinear form $\langle\cdot, \cdot\rangle$ by

$$
\left\langle E_{m, n}, E_{p, q}\right\rangle=\operatorname{tr}\left(E_{m, n} E_{p, q}\right)=\delta_{m, q} \delta_{n, p} \quad \text { for } m, n, p, q \in \mathbb{Z} .
$$

Set $\mathcal{A}=\operatorname{span}\left\{E_{m, n} \mid m+n \in 2 \mathbb{Z}\right\} \subset \mathfrak{g l}_{\infty}$, a subalgebra which is isomorphic to $\mathfrak{g l}_{\infty} \oplus \mathfrak{g l}_{\infty}$. We then have an affine Lie algebra $\widehat{\mathcal{A}}=\mathcal{A} \otimes \mathbb{C}\left[t, t^{-1}\right] \oplus \mathbb{C}$. Let $\sigma$ be the automorphism of $\mathfrak{g l}_{\infty}$ and $\mathcal{A}$ defined by $\sigma\left(E_{m, n}\right)=E_{m+1, n+1}$ for $m, n \in \mathbb{Z}$. It is proved in this paper that $\widehat{A}_{\hbar}$ is isomorphic to the covariant Lie algebra of the affine Lie algebra $\widehat{\mathcal{A}}$ with respect to automorphism group $G=\langle\sigma\rangle$ with linear character $\chi$ given by $\chi\left(\sigma^{k}\right)=e^{i k \hbar}$ for $k \in \mathbb{Z}$. Let $\tau$ be the linear endomorphism of $\mathfrak{g l}_{\infty}$ defined by $\tau\left(E_{m, n}\right)=-E_{n, m}$ for $m, n \in \mathbb{Z}$. Then $\tau$ is an order-2 automorphism of $\mathfrak{g l}_{\infty}$ and $\mathcal{A}$ viewed as Lie algebras. We prove that $\widehat{B}_{\hbar}$ and $\widehat{D}_{\hbar}$ are isomorphic to the covariant Lie algebras of the affine Lie algebra $\widehat{\mathcal{A}}$ with respect to automorphism group $\langle\tau\rangle \times\langle\sigma\rangle$ with suitably defined linear characters. It is also proved that Lie algebras $\widehat{B}_{\hbar}$ and $\widehat{C}_{\hbar}$ are actually isomorphic. Furthermore, we determine irreducible equivariant quasi modules for simple vertex algebra $L_{\widehat{\mathcal{A}}}(\ell, 0)$.

For highest weight modules over an affine Kac-Moody algebra $\widehat{\mathfrak{g}}$, it was known (see $[\mathrm{K}]$ ) that integrability amounts to unitarity. On the other hand, integrability is equivalent to the condition that $e_{\theta}(x)^{\ell+1}=0$ (cf. $\left[\mathrm{LP}\right.$ ), where $e_{\theta}$ is a nonzero root vector of the highest positive long root $\theta$. This equivalent condition plays a very important role in the study of affine vertex operator algebras (cf. [DL], [Li1], [MP1], [MP2]). Here, as a key result we establish an analogous condition that is necessary and sufficient for a restricted $\widehat{A}_{\hbar}$-module of level $\ell$ to be an equivariant quasi $L_{\widehat{\mathcal{A}}}(\ell, 0)$-module. (This condition is similar to the quantum integrability condition studied in [DM], [DF].) Then, by using a result of Kac and Radul (see $[\mathrm{KR}]$ ), we prove that every quasi-finite unitary highest weight (irreducible) $\widehat{A}_{\hbar}$-module of level $\ell$ gives rise to an irreducible equivariant quasi $L_{\widehat{\mathcal{A}}}(\ell, 0)$-module. We conjecture that these are all irreducible equivariant quasi $L_{\widehat{\mathcal{A}}}(\ell, 0)$-modules. 
This paper is organized as follows: In Section 2, we give a realization of trigonometric Lie algebras in terms of the covariant algebras of affine Lie algebras of certain infinitedimensional Lie algebras. In Section 3, we establish an equivalence between the category of restricted $\widehat{A}_{\hbar}$-modules of a level $\ell$ and that of equivariant quasi modules for vertex algebra $V_{\widehat{\mathcal{A}}}(\ell, 0)$. In Section 4 , we study modules for simple vertex algebra $L_{\widehat{\mathcal{A}}}(\ell, 0)$ with $\ell$ a positive integer. In Section 5, we study equivariant quasi modules for $L_{\widehat{\mathcal{A}}}(\ell, 0)$.

\section{Interplay between trigonometric Lie algebras and affine Lie algebras}

In this section, we first recall the trigonometric Lie algebras $\widehat{A}_{\hbar}, \widehat{B}_{\hbar}, \widehat{C}_{\hbar}, \widehat{D}_{\hbar}$, and then give an intrinsic connection of them with the affine Lie algebra $\widehat{\mathcal{A}}$ of a subalgebra $\mathcal{A}$ of the doubly infinite matrix Lie algebra $\mathfrak{g l}_{\infty}$.

We begin by recalling the rank 2 sine Lie algebra (see [FFZ, [G-KL1]).

Definition 2.1. The rank 2 sine Lie algebra $\widehat{A}_{\hbar}$ with a real parameter $\hbar$ is generated by

$$
A_{\alpha, m} \text { for }(\alpha, m) \in \mathbb{Z}^{2},
$$

and by $\mathbf{c}$, a distinguished central element, subject to relations

$$
\left[A_{\alpha, m}, A_{\beta, n}\right]=2 i \sin \hbar(m \beta-n \alpha) A_{\alpha+\beta, m+n}+m \delta_{\alpha+\beta, 0} \delta_{m+n, 0} \mathbf{c}
$$

for $\alpha, \beta, m, n \in \mathbb{Z}$, where $i=\sqrt{-1}$.

Remark 2.2. Note that $A_{0,0}$ was excluded in the original definition. The currently defined $\widehat{A}_{\hbar}$ is a (split) direct sum of the originally defined $\widehat{A}_{\hbar}$ and $\mathbb{C} A_{0,0}$. This slight modification is needed from a vertex-operator point of view, just as with affine Heisenberg Lie algebras.

It can be readily seen that $\widehat{A}_{\hbar}$ is a $\mathbb{Z}$-graded Lie algebra with

$$
\operatorname{deg} \mathbf{c}=0 \text { and } \operatorname{deg} A_{\alpha, m}=m \text { for } \alpha, m \in \mathbb{Z} .
$$

We shall mainly use this $\mathbb{Z}$-grading in this paper. (Note that $\widehat{A}_{\hbar}$ is also a $\mathbb{Z}^{2}$-graded Lie algebra with $\operatorname{deg} A_{\alpha, m}=(\alpha, m)$ for $\alpha, m \in \mathbb{Z}$.) The degree-zero subspace, which is linearly spanned by $\mathbf{c}$ and $A_{\alpha, 0}$ with $\alpha \in \mathbb{Z}$, is an abelian subalgebra. Set

$$
H=\operatorname{span}\left\{A_{\alpha, 0} \mid \alpha \in \mathbb{Z}\right\} .
$$

The notion of $\mathbb{Z}$-graded $\widehat{A}_{\hbar}$-module is defined in the obvious way. A $\mathbb{Z}$-graded $\widehat{A}_{\hbar}$-module is said to be quasifinite (see [KR]) if every homogeneous subspace is finite-dimensional.

An $\widehat{A}_{\hbar}$-module $W$ is said to be of level $\ell \in \mathbb{C}$ if $\mathbf{c}$ acts on $W$ as scalar $\ell$. An $\widehat{A}_{\hbar}$-module $W$ of level $\ell$ is called a highest weight module of highest weight $\lambda \in H^{*}$ if there exists a vector $v \in W$ such that

$$
\begin{aligned}
& A_{\alpha, n} v=0 \quad \text { for all } \alpha, n \in \mathbb{Z} \text { with } n>0, \\
& h v=\lambda(h) v \quad \text { for } h \in H, \\
& W=U\left(\widehat{A}_{\hbar}\right) v .
\end{aligned}
$$


For $\ell \in \mathbb{C}, \lambda \in H^{*}$, denote by $M(\ell, \lambda)$ the generalized Verma module. By definition, $M(\ell, \lambda)$ is the $\widehat{A}_{\hbar}$-module induced from the 1-dimensional module $\mathbb{C}$ for $\widehat{A}_{\bar{\hbar}}^{\geq 0}$, on which $h$ acts as $\lambda(h)$ for $h \in H, \mathbf{c}$ acts as scalar $\ell$, and $\widehat{A}_{\hbar}^{+}$acts trivially. Furthermore, denote by $L(\ell, \lambda)$ the irreducible quotient module of $M(\ell, \lambda)$ by its (unique) maximal submodule.

Set

$$
q=e^{i \hbar}\left(=e^{\hbar \sqrt{-1}}\right) \in \mathbb{C}^{\times} .
$$

For $\alpha \in \mathbb{Z}$, form a generating function

$$
A_{\alpha}(z)=\sum_{n \in \mathbb{Z}} A_{\alpha, n} z^{-n-1} .
$$

Then the defining relations (2.1) can be written as

$$
\begin{aligned}
& {\left[A_{\alpha}(x), A_{\beta}(z)\right]=q^{\alpha} A_{\alpha+\beta}\left(q^{\alpha} z\right) x^{-1} \delta\left(\frac{q^{\alpha+\beta} z}{x}\right)} \\
& \quad-q^{-\alpha} A_{\alpha+\beta}\left(q^{-\alpha} z\right) x^{-1} \delta\left(\frac{q^{-(\alpha+\beta)} z}{x}\right)+\delta_{\alpha+\beta, 0} \frac{\partial}{\partial z} x^{-1} \delta\left(\frac{z}{x}\right) \mathbf{c}
\end{aligned}
$$

for $\alpha, \beta \in \mathbb{Z}$.

Next, we relate $\widehat{A}_{\hbar}$ to an affine Lie algebra. Let $\mathfrak{g}$ be a (possibly infinite-dimensional) Lie algebra equipped with a non-degenerate symmetric invariant bilinear form $\langle\cdot, \cdot\rangle$. Then one has an (untwisted) affine Lie algebra

$$
\hat{\mathfrak{g}}=\mathfrak{g} \otimes \mathbb{C}\left[t, t^{-1}\right] \oplus \mathbb{C} \mathbf{k},
$$

where $\mathbf{k}$ is a nonzero central element and

$$
\left[a \otimes t^{m}, b \otimes t^{n}\right]=[a, b] \otimes t^{m+n}+m \delta_{m+n, 0}\langle a, b\rangle \mathbf{k}
$$

for $a, b \in \mathfrak{g}, m, n \in \mathbb{Z}$.

The following was proved in [Li4] (cf. [G-KK]):

Proposition 2.3. Let $\mathfrak{g}$ be a Lie algebra equipped with a symmetric invariant bilinear form $\langle\cdot, \cdot\rangle$, let $\Gamma$ be a subgroup of $\operatorname{Aut}(\mathfrak{g},\langle\cdot, \cdot\rangle)$, and let $\phi: \Gamma \rightarrow \mathbb{C}^{\times}$be a linear character (group homomorphism). Assume that for $a, b \in \mathfrak{g}$,

$$
[g a, b]=0 \quad \text { and } \quad\langle g a, b\rangle=0 \quad \text { for all but finitely many } g \in \Gamma .
$$

Define a bilinear operation $[\cdot, \cdot]_{\Gamma}$ on the vector space $\mathfrak{g} \otimes \mathbb{C}\left[t, t^{-1}\right] \oplus \mathbb{C} \mathbf{k}$ by

$$
\left[a \otimes t^{m}+\mu \mathbf{k}, b \otimes t^{n}+\nu \mathbf{k}\right]_{\Gamma}=\sum_{g \in \Gamma} \phi(g)^{m}\left([g a, b] \otimes t^{m+n}+m\langle g a, b\rangle \delta_{m+n, 0} \mathbf{k}\right)
$$

for $a, b \in \mathfrak{g}, m, n \in \mathbb{Z}, \mu, \nu \in \mathbb{C}$. Then the subspace linearly spanned by elements

$$
\phi(g)^{m}\left(g a \otimes t^{m}\right)-\left(a \otimes t^{m}\right) \quad \text { for } g \in \Gamma, a \in \mathfrak{g}, m \in \mathbb{Z}
$$

is a two-sided ideal of the nonassociative algebra $\left(\mathfrak{g} \otimes \mathbb{C}\left[t, t^{-1}\right]+\mathbb{C} \mathbf{k},[\cdot, \cdot]_{\Gamma}\right)$, and the quotient algebra, which is denoted by $\hat{\mathfrak{g}}[\Gamma]$, is a Lie algebra. 
The Lie algebra $\hat{\mathfrak{g}}[\Gamma]$ obtained in Proposition 2.3 is called the $(\Gamma, \phi)$-covariant algebra of the affine Lie algebra $\hat{\mathfrak{g}}$, or simply the $\Gamma$-covariant algebra of $\hat{\mathfrak{g}}$ whenever the context is clear. For $a \in \mathfrak{g}, m \in \mathbb{Z}$, we denote by $\overline{a \otimes t^{m}}$ the image of $a \otimes t^{m}$ under the natural map from $\hat{\mathfrak{g}}$ onto $\hat{\mathfrak{g}}[\Gamma]$. We shall still use $\mathbf{k}$ for its image.

Let $\mathfrak{g l}_{\infty}$ be the associative algebra of all doubly infinite complex matrices with only finitely many nonzero entries. Naturally, $\mathfrak{g l}_{\infty}$ is a Lie algebra under the commutator bracket. For $m, n \in \mathbb{Z}$, let $E_{m, n}$ denote the unit matrix whose only nonzero entry is the $(m, n)$-entry which is 1 . Then $E_{m, n}(m, n \in \mathbb{Z})$ form a basis of $\mathfrak{g l}_{\infty}$, where

$$
E_{m, n} \cdot E_{p, q}=\delta_{n, p} E_{m, q}, \quad\left[E_{m, n}, E_{p, q}\right]=\delta_{n, p} E_{m, q}-\delta_{q, m} E_{p, n}
$$

for $m, n, p, q \in \mathbb{Z}$. Equip $\mathfrak{g l}_{\infty}$ with the bilinear form $\langle\cdot, \cdot\rangle$ defined by

$$
\left\langle E_{m, n}, E_{r, s}\right\rangle=\operatorname{tr}\left(E_{m, n} E_{r, s}\right)=\delta_{m, s} \delta_{n, r}
$$

for $m, n, r, s \in \mathbb{Z}$. This bilinear form is non-degenerate, symmetric, and associative.

Definition 2.4. For $r \in \mathbb{Z}$, define a linear operator $\sigma_{r}$ on $\mathfrak{g l}_{\infty}$ by

$$
\sigma_{r}\left(E_{m, n}\right)=E_{m+r, n+r} \quad \text { for } m, n \in \mathbb{Z} .
$$

The following is straightforward to prove (cf. [G-KK]):

Lemma 2.5. The map $\mathbb{Z} \ni n \mapsto \sigma_{n}$ defines a group action of $\mathbb{Z}$ on $\mathfrak{g l}_{\infty}$ by automorphisms which preserve the bilinear form $\langle\cdot, \cdot\rangle$. Furthermore, for any $a, b \in \mathfrak{g l}_{\infty}$, we have

$$
\left[\sigma_{r}(a), b\right]=0 \quad \text { and } \quad\left\langle\sigma_{r}(a), b\right\rangle=0
$$

for all but finitely many $r \in \mathbb{Z}$.

Remark 2.6. As a key ingredient, we here introduce an associative algebra. Set

$$
\mathcal{A}=\operatorname{span}\left\{E_{m, n} \mid m, n \in \mathbb{Z} \text { with } m+n \in 2 \mathbb{Z}\right\},
$$

which is an associative subalgebra of $\mathfrak{g l}_{\infty}$. For $\alpha, m \in \mathbb{Z}$, set

$$
G_{\alpha, m}=E_{\alpha+m, m-\alpha} \in \mathcal{A} \text {. }
$$

Then $G_{\alpha, m}(\alpha, m \in \mathbb{Z})$ form a basis of $\mathcal{A}$ and we have

$$
\begin{aligned}
& G_{\alpha, m} \cdot G_{\beta, n}=\delta_{m-\alpha, n+\beta} G_{\alpha+\beta, \alpha+n}, \\
& \left\langle G_{\alpha, m}, G_{\beta, n}\right\rangle=\delta_{\alpha+\beta, 0} \delta_{m, n} .
\end{aligned}
$$

Furthermore, it can be readily seen that $\mathcal{A}$ is stable under the action of $\mathbb{Z}$, where

$$
\sigma_{r}\left(G_{\alpha, m}\right)=G_{\alpha, m+r} \quad \text { for } r, \alpha, m \in \mathbb{Z} .
$$


View $\mathcal{A}$ as a Lie algebra and equip $\mathcal{A}$ with the symmetric invariant bilinear form $\langle\cdot, \cdot\rangle$ defined above, which is still non-degenerate. Then we have an affine Lie algebra

$$
\widehat{\mathcal{A}}=\mathcal{A} \otimes \mathbb{C}\left[t, t^{-1}\right] \oplus \mathbb{C} \mathbf{k},
$$

on which $\mathbb{Z}$ acts as an automorphism group.

Define a linear character $\chi_{q}: \mathbb{Z} \rightarrow \mathbb{C}^{\times}$by

$$
\chi_{q}(n)=q^{n} \quad \text { for } n \in \mathbb{Z},
$$

recalling that $q=e^{i \hbar}$ where $\hbar$ is the same one as for $\widehat{A}_{\hbar}$. Then we have (cf. [G-KK]):

Proposition 2.7. The sine Lie algebra $\widehat{A}_{\hbar}$ is isomorphic to the $\left(\mathbb{Z}, \chi_{q}\right)$-covariant algebra $\widehat{\mathcal{A}}[\mathbb{Z}]$ of the affine Lie algebra $\widehat{\mathcal{A}}$ with $\mathbf{c}=\mathbf{k}$ and with

$$
A_{\alpha, m}=\overline{G_{\alpha, 0} \otimes t^{m}} \quad \text { for } \alpha, m \in \mathbb{Z} .
$$

Proof. From the definition, $\widehat{A}_{\hbar}$ has a basis $\left\{A_{\alpha, m} \mid \alpha, m \in \mathbb{Z}\right\} \cup\{\mathbf{c}\}$ with the given bracket relations. On the other hand, from Proposition $[2.3, \widehat{\mathcal{A}}[\mathbb{Z}]$ as a vector space is the quotient space of $\widehat{\mathcal{A}}$, modulo the subspace linearly spanned by

$$
\sigma_{n}(a) \otimes t^{m}-q^{-m n}\left(a \otimes t^{m}\right)
$$

for $a \in \mathcal{A}, m, n \in \mathbb{Z}$, and its bracket relation is given by

$$
\left[\overline{a \otimes t^{m}}, \overline{b \otimes t^{n}}\right]=\sum_{r \in \mathbb{Z}} q^{m r}\left(\overline{\left[\sigma_{r}(a), b\right] \otimes t^{m+n}}+m\left\langle\sigma_{r}(a), b\right\rangle \delta_{m+n, 0} \mathbf{k}\right),
$$

where $\overline{u \otimes t^{p}}$ denotes the image of $u \otimes t^{p}$ in $\widehat{\mathcal{A}}[\mathbb{Z}]$ for $u \in \mathcal{A}, p \in \mathbb{Z}$, and $\mathbf{k}$ is identified with its image. We see that $\mathbf{k}$ and $\overline{G_{\alpha, 0} \otimes t^{m}}(\alpha, m \in \mathbb{Z})$ form a basis of $\widehat{\mathcal{A}}[\mathbb{Z}]$. Let $\alpha, \beta, m, n \in \mathbb{Z}$. We have

$$
\begin{aligned}
& {\left[\sigma_{r}\left(G_{\alpha, 0}\right), G_{\beta, 0}\right]=\left[G_{\alpha, r}, G_{\beta, 0}\right]=\delta_{r, \alpha+\beta} G_{\alpha+\beta, \alpha}-\delta_{r,-\alpha-\beta} G_{\alpha+\beta,-\alpha},} \\
& \left\langle\sigma_{r}\left(G_{\alpha, 0}\right), G_{\beta, 0}\right\rangle=\left\langle G_{\alpha, r}, G_{\beta, 0}\right\rangle=\delta_{\alpha+\beta, 0} \delta_{r, 0}
\end{aligned}
$$

for $r \in \mathbb{Z}$. Applying this to (2.21) we get

$$
\begin{aligned}
& {\left[\overline{G_{\alpha, 0} \otimes t^{m}}, \overline{G_{\beta, 0} \otimes t^{n}}\right] } \\
= & q^{m(\alpha+\beta)} \overline{G_{\alpha+\beta, \alpha} \otimes t^{m+n}}-q^{-m(\alpha+\beta)} \overline{G_{\alpha+\beta,-\alpha} \otimes t^{m+n}}+m \delta_{\alpha+\beta, 0} \delta_{m+n, 0} \mathbf{k} \\
= & q^{m \beta-n \alpha} \overline{G_{\alpha+\beta, 0} \otimes t^{m+n}}-q^{n \alpha-m \beta} \overline{G_{\alpha+\beta, 0} \otimes t^{m+n}}+m \delta_{\alpha+\beta, 0} \delta_{m+n, 0} \mathbf{k} \\
= & \left(q^{m \beta-n \alpha}-q^{n \alpha-m \beta}\right) \overline{G_{\alpha+\beta, 0} \otimes t^{m+n}}+m \delta_{\alpha+\beta, 0} \delta_{m+n, 0} \mathbf{k},
\end{aligned}
$$

noticing that

$$
\begin{aligned}
& \overline{G_{\alpha+\beta, \alpha} \otimes t^{m+n}}=\overline{\sigma_{\alpha} G_{\alpha+\beta, 0} \otimes t^{m+n}}=q^{-(m+n) \alpha} \overline{G_{\alpha+\beta, 0} \otimes t^{m+n}} \\
& \overline{G_{\alpha+\beta,-\alpha} \otimes t^{m+n}}=\overline{\sigma_{-\alpha} G_{\alpha+\beta, 0} \otimes t^{m+n}}=q^{(m+n) \alpha} \overline{G_{\alpha+\beta, 0} \otimes t^{m+n}} .
\end{aligned}
$$

Now it follows that $\widehat{A}_{\hbar}$ is isomorphic to $\widehat{\mathcal{A}}[\mathbb{Z}]$ with $A_{\alpha, m}$ corresponding to $\overline{G_{\alpha, 0} \otimes t^{m}}$ for $\alpha, m \in \mathbb{Z}$ and with $\mathbf{c}$ corresponding to $\mathbf{k}$. 
Next, we discuss Lie algebra $\widehat{B}_{\hbar}$. Define a second order automorphism $\tau_{B}$ of $\widehat{A}_{\hbar}$ by

$$
\tau_{B}\left(A_{\alpha, m}\right)=-(-1)^{m} A_{-\alpha, m} \text { for } \alpha, m \in \mathbb{Z} .
$$

Lie algebra $\widehat{B}_{\hbar}$ is defined to be the subalgebra $\left(\widehat{A}_{\hbar}\right)^{\tau_{B}}$ of $\tau_{B}$-fixed points in $\widehat{A}_{\hbar}$, which is linearly spanned by elements

$$
B_{\alpha, m}:=A_{\alpha, m}-(-1)^{m} A_{-\alpha, m}
$$

for $\alpha, m \in \mathbb{Z}$. The commutation relations are

$$
\begin{gathered}
{\left[B_{\alpha, m}, B_{\beta, n}\right]=2 i \sin \hbar(m \beta-n \alpha) B_{\alpha+\beta, m+n}+(-1)^{n} 2 i \sin \hbar(m \beta+n \alpha) B_{\alpha-\beta, m+n}} \\
+2 m\left(\delta_{\alpha+\beta, 0}-(-1)^{m} \delta_{\alpha-\beta, 0}\right) \delta_{m+n, 0} \mathbf{c} .
\end{gathered}
$$

For $\alpha \in \mathbb{Z}$, form a generating function

$$
B_{\alpha}(z)=\sum_{m \in \mathbb{Z}} B_{\alpha, m} z^{-m-1}
$$

That is,

$$
B_{\alpha}(z)=A_{\alpha}(z)+A_{-\alpha}(-z) .
$$

We have

$$
B_{-\alpha}(z)=B_{\alpha}(-z)
$$

for $\alpha \in \mathbb{Z}$, while (2.26) in terms of generating functions becomes

$$
\begin{aligned}
& {\left[B_{\alpha}(x), B_{\beta}(z)\right]=q^{\alpha} B_{\alpha+\beta}\left(q^{\alpha} z\right) x^{-1} \delta\left(\frac{q^{\alpha+\beta} z}{x}\right)-q^{-\alpha} B_{\alpha+\beta}\left(q^{-\alpha} z\right) x^{-1} \delta\left(\frac{q^{-(\alpha+\beta)} z}{x}\right)} \\
& \quad+q^{\alpha} B_{\alpha-\beta}\left(-q^{\alpha} z\right) x^{-1} \delta\left(\frac{-q^{\alpha-\beta} z}{x}\right)-q^{-\alpha} B_{\alpha-\beta}\left(-q^{-\alpha} z\right) x^{-1} \delta\left(\frac{-q^{-(\alpha-\beta)} z}{x}\right) \\
& \quad+2 \delta_{\alpha+\beta, \overline{0}} \frac{\partial}{\partial z} x^{-1} \delta\left(\frac{z}{x}\right) \mathbf{c}-2 \delta_{\alpha-\beta, 0} \frac{\partial}{\partial z} x^{-1} \delta\left(\frac{z}{-x}\right) \mathbf{c} .
\end{aligned}
$$

Definition 2.8. Let $\tau$ be the order-2 automorphism of Lie algebra $\mathfrak{g l}_{\infty}$ defined by

$$
\tau\left(E_{m, n}\right)=-E_{n, m} \quad \text { for } m, n \in \mathbb{Z} .
$$

We summarize some straightforward facts as follows:

Lemma 2.9. The automorphism $\tau$ commutes with the action of $\mathbb{Z}$ on $\mathfrak{g l}_{\infty}$ and also preserves the bilinear form $\langle\cdot, \cdot\rangle$. The group $\mathbb{Z}_{2} \times \mathbb{Z}$ acts on $\mathfrak{g l}_{\infty}$, extending the action of $\mathbb{Z}$ defined in Lemma 2.5, such that

$$
\tau\left(E_{m, n}\right)=-E_{n, m}, \quad \sigma_{r}\left(E_{m, n}\right)=E_{m+r, n+r} \quad \text { for } m, n, r \in \mathbb{Z} .
$$

Furthermore, the subalgebra $\mathcal{A}$ is stable under the action of $\mathbb{Z}_{2} \times \mathbb{Z}$ and we have

$$
\tau\left(G_{\alpha, m}\right)=-G_{-\alpha, m}, \quad \sigma_{r}\left(G_{\alpha, m}\right)=G_{\alpha, m+r} \quad \text { for } \alpha, m, r \in \mathbb{Z} .
$$


Define a linear character $\chi_{q}^{B}: \mathbb{Z}_{2} \times \mathbb{Z} \rightarrow \mathbb{C}^{\times}$by

$$
\chi_{q}^{B}(\tau)=-1 \text { and } \chi_{q}^{B}\left(\sigma_{r}\right)=q^{r} \text { for } r \in \mathbb{Z},
$$

where it is understood that $\mathbb{Z}_{2}=\langle\tau\rangle$ and $\mathbb{Z}=\left\{\sigma_{r} \mid r \in \mathbb{Z}\right\}$. Then we have:

Proposition 2.10. Lie algebra $\widehat{B}_{\hbar}$ is isomorphic to the $\left(\mathbb{Z}_{2} \times \mathbb{Z}, \chi_{q}^{B}\right)$-covariant algebra $\widehat{\mathcal{A}}\left[\mathbb{Z}_{2} \times \mathbb{Z}\right]$ of the affine Lie algebra $\widehat{\mathcal{A}}$ with $\mathbf{c}=\frac{1}{2} \mathbf{k}$ and with

$$
B_{\alpha, m}=\overline{G_{\alpha, 0} \otimes t^{m}} \quad \text { for } \alpha, m \in \mathbb{Z} .
$$

Proof. From the definition, $\widehat{B}_{\hbar}$ is the Lie algebra with generators $B_{\alpha, m}$ for $\alpha, m \in \mathbb{Z}$, subject to relations

$$
\begin{gathered}
B_{-\alpha, m}=-(-1)^{m} B_{\alpha, m} \\
{\left[B_{\alpha, m}, B_{\beta, n}\right]=\left(q^{m \beta-n \alpha}-q^{n \alpha-m \beta}\right) B_{\alpha+\beta, m+n}} \\
+(-1)^{n}\left(q^{m \beta+n \alpha}-q^{-m \beta-n \alpha}\right) B_{\alpha-\beta, m+n}+2 m\left(\delta_{\alpha+\beta, 0}-(-1)^{m} \delta_{\alpha-\beta, 0}\right) \delta_{m+n, 0} \mathbf{c}
\end{gathered}
$$

for $\alpha, \beta, m, n \in \mathbb{Z}$. On the other hand, consider the covariant algebra $\widehat{\mathcal{A}}\left[\mathbb{Z}_{2} \times \mathbb{Z}\right]$ with respect to the linear character $\chi_{q}^{B}$. Let $\alpha, \beta, m, n \in \mathbb{Z}$. From the definition, we have

$$
\begin{aligned}
& {\left[\overline{G_{\alpha, 0} \otimes t^{m}}, \overline{G_{\beta, 0} \otimes t^{n}}\right] } \\
= & \sum_{r \in \mathbb{Z}} q^{m r}\left(\overline{\left[\sigma_{r}\left(G_{\alpha, 0}\right), G_{\beta, 0}\right] \otimes t^{m+n}}+m\left\langle\sigma_{r}\left(G_{\alpha, 0}\right), G_{\beta, 0}\right\rangle \delta_{m+n, 0} \mathbf{k}\right) \\
& +\sum_{r \in \mathbb{Z}}\left(-q^{r}\right)^{m}\left(\overline{\left[\tau \sigma_{r}\left(G_{\alpha, 0}\right), G_{\beta, 0}\right] \otimes t^{m+n}}+m\left\langle\tau \sigma_{r}\left(G_{\alpha, 0}\right), G_{\beta, 0}\right\rangle \delta_{m+n, 0} \mathbf{k}\right) \\
= & \sum_{r \in \mathbb{Z}} q^{m r}\left(\overline{\left[G_{\alpha, r}, G_{\beta, 0}\right] \otimes t^{m+n}}+m\left\langle G_{\alpha, r}, G_{\beta, 0}\right\rangle \delta_{m+n, 0} \mathbf{k}\right) \\
& -\sum_{r \in \mathbb{Z}}\left(-q^{r}\right)^{m}\left(\overline{\left[G_{-\alpha, r}, G_{\beta, 0}\right] \otimes t^{m+n}}+m\left\langle G_{-\alpha, r}, G_{\beta, 0}\right\rangle \delta_{m+n, 0} \mathbf{k}\right) \\
= & \sum_{r \in \mathbb{Z}} q^{m r} \overline{\left[G_{\alpha, r}, G_{\beta, 0}\right] \otimes t^{m+n}}-\sum_{r \in \mathbb{Z}}\left(-q^{r}\right)^{m} \overline{\left[G_{-\alpha, r}, G_{\beta, 0}\right] \otimes t^{m+n}} \\
& +\sum_{r \in \mathbb{Z}} m \overline{\left(q^{m r} \delta_{\alpha+\beta, 0} \delta_{r, 0}-\left(-q^{r}\right)^{m} \delta_{\beta-\alpha, 0} \delta_{r, 0}\right) \delta_{m+n, 0} \mathbf{k}} \\
= & \sum_{r \in \mathbb{Z}} q^{m r} \overline{\left[G_{\alpha, r}, G_{\beta, 0}\right] \otimes t^{m+n}}-\sum_{r \in \mathbb{Z}}\left(-q^{r}\right)^{m} \overline{\left[G_{-\alpha, r}, G_{\beta, 0}\right] \otimes t^{m+n}} \\
& +m\left(\delta_{\alpha+\beta, 0}-(-1)^{m} \delta_{\alpha-\beta, 0}\right) \delta_{m+n, 0} \mathbf{k} .
\end{aligned}
$$

Just as with $\widehat{A}_{\hbar}$ we have

$$
\sum_{r \in \mathbb{Z}} q^{m r} \overline{\left[G_{\alpha, r}, G_{\beta, 0}\right] \otimes t^{m+n}}=\left(q^{m \beta-n \alpha}-q^{n \alpha-m \beta}\right) \overline{G_{\alpha+\beta, 0} \otimes t^{m+n}},
$$


and we also have

$$
\begin{aligned}
& \sum_{r \in \mathbb{Z}}\left(-q^{r}\right)^{m} \overline{\left[G_{-\alpha, r}, G_{\beta, 0}\right] \otimes t^{m+n}} \\
= & \sum_{r \in \mathbb{Z}}\left(-q^{r}\right)^{m}\left(\delta_{r, \beta-\alpha} \overline{G_{\beta-\alpha,-\alpha} \otimes t^{m+n}}-\delta_{r, \alpha-\beta} \overline{G_{\beta-\alpha, \alpha} \otimes t^{m+n}}\right) \\
= & \sum_{r \in \mathbb{Z}}\left(-q^{r}\right)^{m}\left(\delta_{r, \alpha-\beta} \overline{\tau \sigma_{\alpha} G_{\alpha-\beta, 0} \otimes t^{m+n}}-\delta_{r, \beta-\alpha} \overline{\tau \sigma_{-\alpha} G_{\alpha-\beta, 0} \otimes t^{m+n}}\right) \\
= & \sum_{r \in \mathbb{Z}} \delta_{r, \alpha-\beta}\left(-q^{r}\right)^{m}\left(-q^{\alpha}\right)^{-m-n} \overline{G_{\alpha-\beta, 0} \otimes t^{m+n}} \\
& -\sum_{r \in \mathbb{Z}} \delta_{r, \beta-\alpha}\left(-q^{r}\right)^{m}\left(-q^{-\alpha}\right)^{-m-n} \overline{G_{\alpha-\beta, 0} \otimes t^{m+n}} \\
= & (-1)^{n}\left(q^{-m \beta-n \alpha}-q^{m \beta+n \alpha}\right) \overline{G_{\alpha-\beta, 0} \otimes t^{m+n}} .
\end{aligned}
$$

Therefore

$$
\begin{aligned}
& {\left[\overline{G_{\alpha, 0} \otimes t^{m}}, \overline{G_{\beta, 0} \otimes t^{n}}\right] } \\
= & \left(q^{m \beta-n \alpha}-q^{n-m \beta}\right) \overline{G_{\alpha+\beta, 0} \otimes t^{m+n}}+(-1)^{n}\left(q^{m \beta+n \alpha}-q^{-m \beta-n \alpha}\right) \overline{G_{\alpha-\beta, 0} \otimes t^{m+n}} \\
& +m\left(\delta_{\alpha+\beta, 0}-(-1)^{m} \delta_{\alpha-\beta, 0}\right) \delta_{m+n, 0} \mathbf{k} .
\end{aligned}
$$

On the other hand, for $\alpha, m \in \mathbb{Z}$ we have

$$
\overline{G_{-\alpha, 0} \otimes t^{m}}=-\overline{\tau G_{\alpha, 0} \otimes t^{m}}=-\chi(\tau)^{-m} \overline{G_{\alpha, 0} \otimes t^{m}}=-(-1)^{m} \overline{G_{\alpha, 0} \otimes t^{m}} .
$$

Therefore, $\widehat{B}_{\hbar}$ is isomorphic to $\widehat{\mathcal{A}}\left[\mathbb{Z}_{2} \times \mathbb{Z}\right]$ with $B_{\alpha, m}$ corresponding to $\overline{G_{\alpha, 0} \otimes t^{m}}$ for $\alpha, m \in \mathbb{Z}$ and with $\mathbf{c}$ corresponding to $\frac{1}{2} \mathbf{k}$.

The Lie algebra $\widehat{C}_{\hbar}$ is the subalgebra of $\tau_{C}$-fixed points in $\widehat{A}_{\hbar}$, where $\tau_{C}$ is the second order automorphism of $\widehat{A}_{\hbar}$, defined by

$$
\tau_{C}\left(A_{\alpha, m}\right)=-(-1)^{m} q^{2 \alpha} A_{-\alpha, m} \quad \text { for } \alpha, m \in \mathbb{Z} .
$$

As a subspace, $\widehat{C}_{\hbar}$ is linearly spanned by

$$
C_{\alpha, m}:=A_{\alpha, m}-(-1)^{m} q^{2 \alpha} A_{-\alpha, m} \quad \text { for } \alpha, m \in \mathbb{Z},
$$

where the commutation relations are

$$
\begin{aligned}
{\left[C_{\alpha, m}, C_{\beta, n}\right]=} & 2 i \sin \hbar(m \beta-n \alpha) C_{\alpha+\beta, m+n} \\
& +(-1)^{n} 2 i q^{2 \beta} \sin \hbar(m \beta+n \alpha) C_{\alpha-\beta, m+n} \\
& +2 m\left(\delta_{\alpha+\beta, 0}-(-1)^{m} q^{2 \alpha} \delta_{\alpha-\beta, 0}\right) \delta_{m+n, 0} \mathbf{c} .
\end{aligned}
$$

In terms of generating functions we have

$$
C_{\alpha}(x)=A_{\alpha}(x)+q^{2 \alpha} A_{-\alpha}(-x) .
$$

Note that

$$
C_{-\alpha}(x)=A_{-\alpha}(x)+q^{-2 \alpha} A_{\alpha}(-x)=q^{-2 \alpha} C_{\alpha}(-x) .
$$

We have: 
Proposition 2.11. Lie algebra $\widehat{C}_{\hbar}$ is isomorphic to $\widehat{B}_{\hbar}$, which is isomorphic to the $\left(\mathbb{Z}_{2} \times\right.$ $\left.\mathbb{Z}, \chi_{q}^{B}\right)$-covariant algebra $\widehat{\mathcal{A}}\left[\mathbb{Z}_{2} \times \mathbb{Z}\right]$.

Proof. For $\alpha \in \mathbb{Z}$, set

$$
C_{\alpha}^{\prime}(x)=q^{-\alpha} C_{\alpha}(x)=q^{-\alpha} A_{\alpha}(x)+q^{\alpha} A_{-\alpha}(-x) .
$$

Then we have

$$
C_{-\alpha}^{\prime}(x)=C_{\alpha}^{\prime}(-x)
$$

and

$$
\begin{aligned}
{\left[C_{\alpha, m}^{\prime}, C_{\beta, n}^{\prime}\right]=} & 2 i \sin \hbar(m \beta-n \alpha) C_{\alpha+\beta, m+n}^{\prime} \\
& +(-1)^{n} 2 i \sin \hbar(m \beta+n \alpha) C_{\alpha-\beta, m+n}^{\prime} \\
& +2 m\left(\delta_{\alpha+\beta, 0}-(-1)^{m} \delta_{\alpha-\beta, 0}\right) \delta_{m+n, 0} \mathbf{c} .
\end{aligned}
$$

Comparing these with (2.29) and (2.30), we conclude that $\widehat{C}_{\hbar}$ is isomorphic to $\widehat{B}_{\hbar}$ with $C_{\alpha}^{\prime}(x)$ corresponding to $B_{\alpha}(x)$ for $\alpha \in \mathbb{Z}$ and with $\mathbf{c}$ corresponding to $\mathbf{c}$.

Last, we discuss the Lie algebra $\widehat{D}_{\hbar}$. Let $\tau_{D}$ be the linear endomorphism of $\widehat{A}_{\hbar}$ defined by

$$
\tau_{D}(\mathbf{c})=\mathbf{c}, \quad \tau_{D}\left(A_{\alpha, m}\right)=-q^{2 \alpha} A_{-\alpha, m} \quad \text { for } \alpha, m \in \mathbb{Z} .
$$

It is straightforward to show that $\tau_{D}$ is an order-2 automorphism of $\widehat{A}_{\hbar}$. Lie algebra $\widehat{D}_{\hbar}$ is defined to be the $\tau_{D}$-fixed points subalgebra of $A_{\hbar}$.

For $\alpha, m \in \mathbb{Z}$, set

$$
D_{\alpha, m}=A_{\alpha, m}-q^{2 \alpha} A_{-\alpha, m}
$$

Then $\widehat{D}_{\hbar}$ is linearly spanned by $D_{\alpha, m}$ for $\alpha, m \in \mathbb{Z}$. We have

$$
D_{-\alpha, m}=-q^{-2 \alpha} D_{\alpha, m} \quad \text { for } \alpha, m \in \mathbb{Z}
$$

and

$$
\begin{aligned}
& {\left[D_{\alpha, m}, D_{\beta, n}\right]=2 i \sin \hbar(m \beta-n \alpha) D_{\alpha+\beta, m+n}} \\
& \quad+2 i q^{2 \beta} \sin \hbar(m \beta+n \alpha) D_{\alpha-\beta, m+n}+2 m\left(\delta_{\alpha+\beta, 0}-q^{2 \alpha} \delta_{\alpha-\beta, 0}\right) \delta_{m+n, 0} \cdot \mathbf{c} .
\end{aligned}
$$

For $\alpha, m \in \mathbb{Z}$, set

$$
D_{\alpha}(x)=\sum_{m \in \mathbb{Z}} D_{\alpha, m} x^{-m-1}
$$


In terms of generating functions, we have

$$
\begin{aligned}
& {\left[D_{\alpha}(x), D_{\beta}(z)\right]=q^{\alpha} D_{\alpha+\beta}\left(q^{\alpha} z\right) x^{-1} \delta\left(\frac{q^{\alpha+\beta} z}{x}\right)-q^{-\alpha} D_{\alpha+\beta}\left(q^{-\alpha} z\right) x^{-1} \delta\left(\frac{q^{-(\alpha+\beta)} z}{x}\right)} \\
& \quad+q^{2 \beta-\alpha} D_{\alpha-\beta}\left(q^{-\alpha} z\right) x^{-1} \delta\left(\frac{q^{-(\alpha-\beta)} z}{x}\right)-q^{\alpha+2 \beta} D_{\alpha-\beta}\left(q^{\alpha} z\right) x^{-1} \delta\left(\frac{q^{\alpha-\beta} z}{x}\right) \\
& \quad+2\left(\delta_{\alpha+\beta, 0}-q^{2 \alpha} \delta_{\alpha-\beta, 0}\right) \frac{\partial}{\partial z} x^{-1} \delta\left(\frac{z}{x}\right) \cdot \mathbf{c} .
\end{aligned}
$$

Define a linear character $\chi_{q}^{D}: \mathbb{Z}_{2} \times \mathbb{Z} \rightarrow \mathbb{C}^{\times}$by

$$
\chi_{q}^{D}(\tau)=1 \text { and } \chi_{q}^{D}\left(\sigma_{r}\right)=q^{r} \text { for } r \in \mathbb{Z}
$$

Now, we have:

Proposition 2.12. Lie algebra $\widehat{D}_{\hbar}$ is isomorphic to the $\left(\mathbb{Z}_{2} \times \mathbb{Z}, \chi_{q}^{D}\right)$-covariant algebra of affine Lie algebra $\widehat{\mathcal{A}}$ with $c=\frac{1}{2} \mathbf{k}$ and

$$
D_{\alpha, m}=q^{\alpha} \overline{G_{\alpha, 0} \otimes t^{m}} \quad \text { for } \alpha, m \in \mathbb{Z} \text {. }
$$

Proof. For $\alpha, m \in \mathbb{Z}$, set $D_{\alpha, m}^{\prime}=q^{-\alpha} D_{\alpha, m}$. Then we have

$$
D_{-\alpha, m}^{\prime}=-D_{\alpha, m}^{\prime}
$$

and

$$
\begin{aligned}
& {\left[D_{\alpha, m}^{\prime}, D_{\beta, n}^{\prime}\right]=\left(q^{m \beta-n \alpha}-q^{n \alpha-m \beta}\right) D_{\alpha+\beta, m+n}^{\prime}} \\
& \quad+\left(q^{m \beta+n \alpha}-q^{-m \beta-n \alpha}\right) D_{\alpha-\beta, m+n}^{\prime}+2 m\left(\delta_{\alpha+\beta, 0}-\delta_{\alpha-\beta, 0}\right) \delta_{m+n, 0} \cdot \mathbf{c}
\end{aligned}
$$

for $\alpha, \beta, m, n \in \mathbb{Z}$. We see that $\widehat{D}_{\hbar}$ can be defined alternatively as the Lie algebra with generators $D_{\alpha, m}^{\prime}$ for $\alpha, m \in \mathbb{Z}$, subject to these relations.

On the other hand, consider the covariant algebra $\widehat{\mathcal{A}}\left[\mathbb{Z}_{2} \times \mathbb{Z}\right]$ with respect to the linear character $\chi_{q}^{D}$. Let $\alpha, \beta, r, m, n \in \mathbb{Z}$. Just as with $\hat{A}_{\hbar}$, we have

$$
\sum_{r \in \mathbb{Z}} \chi_{q}^{D}\left(\sigma_{r}\right)^{m} \overline{\left[\sigma_{r} G_{\alpha, 0}, G_{\beta, 0}\right] \otimes t^{m+n}}=\left(q^{m \beta-n \alpha}-q^{n \alpha-m \beta}\right) \overline{G_{\alpha+\beta, 0} \otimes t^{m+n}} .
$$


As $\chi_{q}^{D}\left(\tau \sigma_{r}\right)=q^{r}$ for $r \in \mathbb{Z}$, we have

$$
\begin{aligned}
& \sum_{r \in \mathbb{Z}} \chi_{q}^{D}\left(\tau \sigma_{r}\right)^{m} \overline{\left[\tau \sigma_{r} G_{\alpha, 0}, G_{\beta, 0}\right] \otimes t^{m+n}} \\
= & -\sum_{r \in \mathbb{Z}} q^{r m} \overline{\left[G_{-\alpha, r}, G_{\beta, 0}\right] \otimes t^{m+n}} \\
= & -\sum_{r \in \mathbb{Z}} q^{r m}\left(\delta_{r, \beta-\alpha} \overline{G_{\beta-\alpha,-\alpha} \otimes t^{m+n}}-\delta_{r, \alpha-\beta} \overline{G_{\beta-\alpha, \alpha} \otimes t^{m+n}}\right) \\
= & \sum_{r \in \mathbb{Z}} q^{r m}\left(\delta_{r, \beta-\alpha} \overline{\tau \sigma \sigma_{-\alpha} G_{\alpha-\beta, 0} \otimes t^{m+n}}-\delta_{r, \alpha-\beta} \overline{\tau \sigma_{\alpha} G_{\alpha-\beta, 0} \otimes t^{m+n}}\right) \\
= & \sum_{r \in \mathbb{Z}}\left(\delta_{r, \beta-\alpha} q^{r m} q^{\alpha(m+n)} \overline{G_{\alpha-\beta, 0} \otimes t^{m+n}}-\delta_{r, \alpha-\beta} q^{r m} q^{-\alpha(m+n)} \overline{G_{\alpha-\beta, 0} \otimes t^{m+n}}\right) \\
= & \left(q^{m \beta+n \alpha}-q^{-m \beta-n \alpha}\right) \overline{G_{\alpha-\beta, 0} \otimes t^{m+n}} .
\end{aligned}
$$

We also have

$$
\begin{aligned}
& \chi_{q}^{D}\left(\sigma_{r}\right)^{m}\left\langle\sigma_{r} G_{\alpha, 0}, G_{\beta, 0}\right\rangle=q^{r m}\left\langle G_{\alpha, r}, G_{\beta, 0}\right\rangle=q^{r m} \delta_{\alpha+\beta, 0} \delta_{r, 0}=\delta_{\alpha+\beta, 0} \delta_{r, 0}, \\
& \chi_{q}^{D}\left(\tau \sigma_{r}\right)^{m}\left\langle\tau \sigma_{r} G_{\alpha, 0}, G_{\beta, 0}\right\rangle=-q^{r m}\left\langle G_{-\alpha, r}, G_{\beta, 0}\right\rangle=-q^{r m} \delta_{\beta-\alpha, 0} \delta_{r, 0}=-\delta_{\alpha-\beta, 0} \delta_{r, 0} .
\end{aligned}
$$

Using all these relations we get

$$
\begin{aligned}
& {\left[\overline{G_{\alpha, 0} \otimes t^{m}}, \overline{G_{\beta, 0} \otimes t^{n}}\right]=\left(q^{m \beta-n \alpha}-q^{n \alpha-m \beta}\right) \overline{G_{\alpha+\beta, 0} \otimes t^{m+n}}} \\
& \quad+\left(q^{m \beta+n \alpha}-q^{-m \beta-n \alpha}\right) \overline{G_{\alpha-\beta, 0} \otimes t^{m+n}}+m\left(\delta_{\alpha+\beta, 0}-\delta_{\alpha-\beta, 0}\right) \delta_{m+n, 0} \mathbf{k} .
\end{aligned}
$$

Furthermore, for $\alpha, m \in \mathbb{Z}$ we have

$$
\overline{G_{-\alpha, 0} \otimes t^{m}}=-\overline{\tau G_{\alpha, 0} \otimes t^{m}}=-\chi_{q}^{D}(\tau)^{-n} \overline{G_{\alpha, 0} \otimes t^{m}}=-\overline{G_{\alpha, 0} \otimes t^{m}} .
$$

Now, the assertion follows.

Recall from Definition 2.8 the order-2 automorphism $\tau$ of $\mathfrak{g l}_{\infty}$, which preserves $\mathcal{A}$. Denote by $\mathcal{A}^{\tau}$ the Lie subalgebra of $\tau$-fixed points in $\mathcal{A}$ :

$$
\mathcal{A}^{\tau}=\{a \in \mathcal{A} \mid \tau(a)=a\}
$$

For $\alpha, m \in \mathbb{Z}$, set

$$
G_{\alpha, m}^{\tau}=G_{\alpha, m}-G_{-\alpha, m} \in \mathcal{A}^{\tau}
$$

Note that $G_{\alpha, m}^{\tau}$ for $\alpha \geq 1, m \in \mathbb{Z}$ form a basis of $\mathcal{A}^{\tau}$. Using (2.16) we get

$$
\begin{aligned}
& {\left[G_{\alpha, m}^{\tau}, G_{\beta, n}^{\tau}\right]=\delta_{m-\alpha, n+\beta} G_{\alpha+\beta, n+\alpha}^{\tau}-\delta_{m+\alpha, n-\beta} G_{\alpha+\beta, \beta+m}^{\tau}} \\
& +\delta_{m+\alpha, n+\beta} G_{\alpha-\beta, m-\beta}^{\tau}-\delta_{m-\alpha, n-\beta} G_{\alpha-\beta, \alpha+n}^{\tau}, \\
& \left\langle G_{\alpha, m}^{\tau}, G_{\beta, n}^{\tau}\right\rangle=2\left(\delta_{\alpha+\beta, 0}-\delta_{\alpha-\beta, 0}\right) \delta_{m, n}
\end{aligned}
$$

for $\alpha, \beta, m, n \in \mathbb{Z}$. We have the following variation of Proposition 2.12 : 
Proposition 2.13. Lie algebra $\widehat{D}_{\hbar}$ is isomorphic to the covariant algebra $\widehat{\mathcal{A}^{\tau}}[\mathbb{Z}]$ with

$$
D_{\alpha, m}=q^{\alpha} \overline{G_{\alpha, 0}^{\tau} \otimes t^{m}} \quad \text { for } \alpha, m \in \mathbb{Z}
$$

and with $\mathbf{c}=\mathbf{k}$.

Proof. It is similar to the proof of Proposition 2.12. Notice that $\mathbf{c}$ and $D_{\alpha, m}^{\prime}$ for $\alpha \geq 1, m \in$ $\mathbb{Z}$ form a basis of $\widehat{D}_{\hbar}$, where $D_{\alpha, m}^{\prime}=q^{-\alpha} D_{\alpha, m}$. On the other hand, $\mathbf{k}$ and $\overline{G_{\alpha, 0}^{\tau} \otimes t^{m}}$ for $\alpha \geq 1, m \in \mathbb{Z}$ form a basis of $\widehat{\mathcal{A}^{\tau}}[\mathbb{Z}]$. Then we have a linear isomorphism $\theta: \widehat{D}_{\hbar} \rightarrow \widehat{\mathcal{A}^{\tau}}[\mathbb{Z}]$ such that $\theta(\mathbf{c})=\mathbf{k}$ and

$$
\theta\left(D_{\alpha, m}\right)=q^{\alpha} \overline{\left(G_{\alpha, 0}^{\tau} \otimes t^{m}\right)} \quad \text { for } \alpha, m \in \mathbb{Z},
$$

noticing that $D_{-\alpha, m}^{\prime}=-D_{\alpha, m}^{\prime}$ in $\widehat{D}_{\hbar}$ and $\overline{G_{-\alpha, 0}^{\tau} \otimes t^{n}}=-\overline{G_{\alpha, 0}^{\tau} \otimes t^{n}}$ in $\widehat{\mathcal{A}^{\tau}}[\mathbb{Z}]$. Let $\alpha, \beta, m, n \in$ $\mathbb{Z}$. As $\sigma_{r}\left(G_{\alpha, 0}^{\tau}\right)=G_{\alpha, r}^{\tau}$ and $\chi_{q}\left(\sigma_{r}\right)=q^{r}$ for $r \in \mathbb{Z}$, from Proposition 2.3 we have

$$
\begin{aligned}
& {\left[G_{\alpha, 0}^{\tau} \otimes t^{m}, G_{\beta, 0}^{\tau} \otimes t^{n}\right]_{\mathbb{Z}} } \\
& \sum_{r \in \mathbb{Z}} q^{r m}\left(\left[G_{\alpha, r}^{\tau}, G_{\beta, 0}^{\tau}\right] \otimes t^{m+n}+m \delta_{m+n, 0}\left\langle G_{\alpha, r}^{\tau}, G_{\beta, 0}^{\tau}\right\rangle \mathbf{k}\right) .
\end{aligned}
$$

Furthermore, we have

$$
\begin{aligned}
& \sum_{r \in \mathbb{Z}} q^{r m} \overline{\left[G_{\alpha, r}^{\tau}, G_{\beta, 0}^{\tau}\right] \otimes t^{m+n}} \\
= & \sum_{r \in \mathbb{Z}} q^{r m} \overline{\left(\delta_{r, \alpha+\beta} G_{\alpha+\beta, \alpha}^{\tau}-\delta_{r,-\alpha-\beta} G_{\alpha+\beta,-\alpha}^{\tau}+\delta_{r, \beta-\alpha} G_{\alpha-\beta,-\alpha}^{\tau}-\delta_{r, \alpha-\beta} G_{\alpha-\beta, \alpha}^{\tau}\right) \otimes t^{m+n}} \\
= & \overline{\left(q^{m(\alpha+\beta)} G_{\alpha+\beta, \alpha}^{\tau}-q^{-m(\alpha+\beta)} G_{\alpha+\beta,-\alpha}^{\tau}+q^{m(\beta-\alpha)} G_{\alpha-\beta,-\alpha}^{\tau}-q^{m(\alpha-\beta)} G_{\alpha-\beta, \alpha}^{\tau}\right) \otimes t^{m+n}} \\
= & \overline{\left(q^{m(\alpha+\beta)} \sigma_{\alpha} G_{\alpha+\beta, 0}^{\tau}-q^{-m(\alpha+\beta)} \sigma_{-\alpha} G_{\alpha+\beta, 0}^{\tau}\right) \otimes t^{m+n}} \\
& +\overline{\left(q^{m(\beta-\alpha)} \sigma_{-\alpha} G_{\alpha-\beta, 0}^{\tau}-q^{m(\alpha-\beta)} \sigma_{\alpha} G_{\alpha-\beta, 0}^{\tau}\right) \otimes t^{m+n}} \\
= & \left(q^{m \beta-n \alpha}-q^{n \alpha-m \beta}\right) \overline{G_{\alpha+\beta, 0}^{\tau} \otimes t^{m+n}}+\left(q^{m \beta+n \alpha}-q^{-m \beta-n \alpha}\right) \overline{G_{\alpha-\beta, 0} \otimes t^{m+n}}
\end{aligned}
$$

and

$$
\sum_{r \in \mathbb{Z}}\left\langle G_{\alpha, r}^{\tau}, G_{\beta, 0}^{\tau}\right\rangle=\sum_{r \in \mathbb{Z}} 2\left(\delta_{\alpha+\beta, 0}-\delta_{\alpha-\beta, 0}\right) \delta_{r, 0}=2\left(\delta_{\alpha+\beta, 0}-\delta_{\alpha-\beta, 0}\right) .
$$

Then we obtain

$$
\begin{aligned}
& {\left[\overline{G_{\alpha, 0}^{\tau} \otimes t^{m}}, \overline{G_{\beta, 0}^{\tau} \otimes t^{n}}\right] } \\
= & \left(q^{m \beta-n \alpha}-q^{n \alpha-m \beta}\right) \overline{G_{\alpha+\beta, 0}^{\tau} \otimes t^{m+n}}+\left(q^{m \beta+n \alpha}-q^{-m \beta-n \alpha}\right) \overline{G_{\alpha-\beta, 0} \otimes t^{m+n}} \\
& +2 m \delta_{m+n, 0}\left(\delta_{\alpha+\beta, 0}-\delta_{\alpha-\beta, 0}\right) \mathbf{k} .
\end{aligned}
$$

It now follows from this and (2.51) that $\theta$ is a Lie algebra isomorphism. 


\section{Interplay between modules for trigonometric Lie algebras and quasi modules for vertex algebras}

In this section, we first recall from [Li3] (cf. [Li4]) the notion of vertex $\Gamma$-algebra and the notion of equivariant quasi module for a vertex $\Gamma$-algebra, and then as the main results, we show that restricted modules of level $\ell$ for trigonometric Lie algebras are equivariant quasi modules for the vertex algebra $V_{\widehat{\mathcal{A}}}(\ell, 0)$ with $\Gamma$ equal to the automorphism group $\mathbb{Z}$ or $\mathbb{Z}_{2} \times \mathbb{Z}$.

We begin by recalling from [Li3] the notion of quasi module for a vertex algebra.

Definition 3.1. Let $V$ be a vertex algebra. A quasi $V$-module is a vector space $W$ equipped with a linear map

$$
\begin{aligned}
Y_{W}(\cdot, x): \quad & V \rightarrow \operatorname{Hom}(W, W((x))) \subset(\operatorname{End} W)\left[\left[x, x^{-1}\right]\right] \\
& v \mapsto Y_{W}(v, x)
\end{aligned}
$$

satisfying the conditions that $Y_{W}(\mathbf{1}, x)=1_{W}$ (the identity operator on $W$ ) and that for $u, v \in V$, there exists a nonzero polynomial $p\left(x_{1}, x_{2}\right)$ such that

$$
\begin{aligned}
x_{0}^{-1} \delta & \left(\frac{x_{1}-x_{2}}{x_{0}}\right) p\left(x_{1}, x_{2}\right) Y_{W}\left(u, x_{1}\right) Y_{W}\left(v, x_{2}\right) \\
& \quad-x_{0}^{-1} \delta\left(\frac{x_{2}-x_{1}}{-x_{0}}\right) p\left(x_{1}, x_{2}\right) Y_{W}\left(v, x_{2}\right) Y_{W}\left(u, x_{1}\right) \\
= & x_{2}^{-1} \delta\left(\frac{x_{1}-x_{0}}{x_{2}}\right) p\left(x_{1}, x_{2}\right) Y_{W}\left(Y\left(u, x_{0}\right) v, x_{2}\right)
\end{aligned}
$$

(the quasi Jacobi identity).

It is clear that the notion of quasi module generalizes that of module. In the definition of a module, the Jacobi identity can be replaced by weak associativity (cf. [Li1], [LL]). The following is an analog for quasi modules, whose proof is straightforward (cf. [LTW]):

Lemma 3.2. Let $V$ be a vertex algebra. In the definition of a quasi $V$-module, the quasi Jacobi identity can be equivalently replaced with the property that for $u, v \in V$, there exists a nonzero polynomial $q\left(x_{1}, x_{2}\right)$ such that

$$
q\left(x_{1}, x_{2}\right) Y_{W}\left(u, x_{1}\right) Y_{W}\left(v, x_{2}\right) \in \operatorname{Hom}\left(W, W\left(\left(x_{1}, x_{2}\right)\right)\right)
$$

and

$$
q\left(x_{2}+x_{0}, x_{2}\right) Y_{W}\left(Y\left(u, x_{0}\right) v, x_{2}\right)=\left.\left(q\left(x_{1}, x_{2}\right) Y_{W}\left(u, x_{1}\right) Y_{W}\left(v, x_{2}\right)\right)\right|_{x_{1}=x_{2}+x_{0}} .
$$

The following is a simple fact that we frequently use: 
Lemma 3.3. Let $V$ be a vertex algebra and $\left(W, Y_{W}\right)$ a quasi $V$-module. For $u, v \in V$, if

$$
h\left(x_{1}, x_{2}\right) Y_{W}\left(u, x_{1}\right) Y_{W}\left(v, x_{2}\right) \in \operatorname{Hom}\left(W, W\left(\left(x_{1}, x_{2}\right)\right)\right)
$$

for some series $h\left(x_{1}, x_{2}\right) \in \mathbb{C}\left(\left(x_{1}, x_{2}\right)\right)$, then

$$
h\left(x_{2}+x_{0}, x_{2}\right) Y_{W}\left(Y\left(u, x_{0}\right) v, x_{2}\right)=\left.\left(h\left(x_{1}, x_{2}\right) Y_{W}\left(u, x_{1}\right) Y_{W}\left(v, x_{2}\right)\right)\right|_{x_{1}=x_{2}+x_{0}} .
$$

Proof. Let $q\left(x_{1}, x_{2}\right)$ be a nonzero polynomial such that (3.2) and (3.3) hold. Then

$$
\begin{aligned}
& h\left(x_{2}+x_{0}, x_{2}\right) q\left(x_{2}+x_{0}, x_{2}\right) Y_{W}\left(Y\left(u, x_{0}\right) v, x_{2}\right) \\
= & \left.\left(h\left(x_{1}, x_{2}\right) q\left(x_{1}, x_{2}\right) Y_{W}\left(u, x_{1}\right) Y_{W}\left(v, x_{2}\right)\right)\right|_{x_{1}=x_{2}+x_{0}} \\
= & \left.q\left(x_{2}+x_{0}, x_{2}\right)\left(h\left(x_{1}, x_{2}\right) Y_{W}\left(u, x_{1}\right) Y_{W}\left(v, x_{2}\right)\right)\right|_{x_{1}=x_{2}+x_{0}} .
\end{aligned}
$$

Notice that we are allowed to cancel the factor $q\left(x_{2}+x_{0}, x_{0}\right)$ (a nonzero element of $\left.\mathbb{C}\left(\left(x_{2}\right)\right)\left(\left(x_{0}\right)\right)\right)$, and by doing so we obtain (3.4).

The following notion of vertex $\Gamma$-algebra was introduced in [Li3]:

Definition 3.4. Let $\Gamma$ be a group. A vertex $\Gamma$-algebra is a vertex algebra $V$ equipped with two group homomorphisms

$$
\begin{aligned}
& R: \Gamma \rightarrow \mathrm{GL}(V) ; \quad g \mapsto R_{g} \\
& \phi: \Gamma \rightarrow \mathbb{C}^{\times},
\end{aligned}
$$

satisfying the condition that $R_{g}(\mathbf{1})=\mathbf{1}$,

$$
R_{g} Y(v, x)=Y\left(R_{g}(v), \phi(g)^{-1} x\right) R_{g} \quad \text { for } g \in \Gamma, v \in V .
$$

Remark 3.5. Let $V$ be a $\mathbb{Z}$-graded vertex algebra in the sense that $V$ is a vertex algebra equipped with a $\mathbb{Z}$-grading $V=\oplus_{n \in \mathbb{Z}} V_{(n)}$ such that $\mathbf{1} \in V_{(0)}$ and

$$
u_{m} V_{(n)} \subset V_{(n+k-m-1)} \quad \text { for } u \in V_{(k)}, m, n, k \in \mathbb{Z} .
$$

Define a linear operator $L(0)$ on $V$ by $L(0) v=n v$ for $v \in V_{(n)}$ with $n \in \mathbb{Z}$. Suppose that $\Gamma$ is a group of automorphisms of $V$ that preserve the $\mathbb{Z}$-grading. Let $\phi: \Gamma \rightarrow \mathbb{C}^{\times}$be any linear character. For $g \in \Gamma$, set $R_{g}=\phi(g)^{-L(0)} g$. Then $V$ becomes a vertex $\Gamma$-algebra (see [Li3]).

Definition 3.6. Let $V$ be a vertex $\Gamma$-algebra. An equivariant quasi $V$-module is a quasi module $\left(W, Y_{W}\right)$ for $V$ viewed as a vertex algebra, satisfying the conditions that

$$
Y_{W}\left(R_{g} v, x\right)=Y_{W}(v, \phi(g) x) \quad \text { for } g \in \Gamma, v \in V
$$

and that for $u, v \in V$, (3.1) holds with some polynomial $p\left(x_{1}, x_{2}\right)$ of the form

$$
p\left(x_{1}, x_{2}\right)=\left(x_{1}-\phi\left(g_{1}\right) x_{2}\right) \cdots\left(x_{1}-\phi\left(g_{k}\right) x_{2}\right)
$$

for some (not necessarily distinct) $g_{1}, \ldots, g_{k} \in \Gamma$. 
To emphasize the dependence on the group $\Gamma$ and the linear character $\chi$, we shall also use the term $(\Gamma, \chi)$-equivariant quasi $V$-module.

The following result was obtained in [Li4] (Proposition 2.13):

Proposition 3.7. Let $V$ be a vertex $\Gamma$-algebra, let $\psi: \phi(\Gamma) \rightarrow \Gamma$ be a section of the linear character $\phi: \Gamma \rightarrow \mathbb{C}^{\times}$, and let $\left(W, Y_{W}\right)$ be an equivariant quasi $V$-module. For $u, v \in V$, we have

$$
\begin{aligned}
& {\left[Y_{W}\left(u, x_{1}\right), Y_{W}\left(v, x_{2}\right)\right] } \\
= & \sum_{\alpha \in \phi(\Gamma)} \operatorname{Res}_{x_{0}} x_{1}^{-1} \delta\left(\frac{\alpha x_{2}+x_{0}}{x_{1}}\right) Y_{W}\left(Y\left(R_{\psi(\alpha)} u, \alpha^{-1} x_{0}\right) v, x_{2}\right),
\end{aligned}
$$

which is a finite sum.

As an immediate consequence we have:

Corollary 3.8. Under all the assumptions in Proposition 3.7, let $u, v \in V$. Set

$$
S_{u, v}=\left\{g \in \operatorname{Im}(\psi) \subset \Gamma \mid(g u)_{n} v \neq 0 \quad \text { for some } n \geq 0\right\} .
$$

For $g \in S_{u, v}$, let $k_{g} \in \mathbb{N}$ be such that $(g u)_{m} v=0$ for $m \geq k_{g}$. Then there are finitely many $g_{1}, \ldots, g_{r} \in S_{u, v}$ such that

$$
\left(\prod_{i=1}^{r}\left(x_{1}-\phi\left(g_{i}\right) x_{2}\right)^{k_{g_{i}}}\right)\left[Y_{W}\left(u, x_{1}\right), Y_{W}\left(v, x_{2}\right)\right]=0
$$

Now, consider a (general) affine Lie algebra $\hat{\mathfrak{g}}$. For $a \in \mathfrak{g}$, form a generating function

$$
a(x)=\sum_{n \in \mathbb{Z}} a(n) x^{-n-1} \in \hat{\mathfrak{g}}\left[\left[x, x^{-1}\right]\right],
$$

where $a(n)$ stands for $a \otimes t^{n}$. The defining relations (2.6) can be written as

$$
\left[a\left(x_{1}\right), b\left(x_{2}\right)\right]=[a, b]\left(x_{2}\right) x_{1}^{-1} \delta\left(\frac{x_{2}}{x_{1}}\right)+\langle a, b\rangle \frac{\partial}{\partial x_{2}} x_{1}^{-1} \delta\left(\frac{x_{2}}{x_{1}}\right) \mathbf{k}
$$

for $a, b \in \mathfrak{g}$. Let $\ell$ be a complex number. View $\mathbb{C}$ as a module for subalgebra $\mathfrak{g} \otimes \mathbb{C}[t]+\mathbb{C} \mathbf{k}$ with $\mathfrak{g} \otimes \mathbb{C}[t]$ acting trivially and with $\mathbf{k}$ acting as scalar $\ell$. Then form an induced module

$$
V_{\hat{\mathfrak{g}}}(\ell, 0)=U(\hat{\mathfrak{g}}) \otimes_{U(\mathfrak{g} \otimes \mathbb{C}[t]+\mathbb{C k})} \mathbb{C} .
$$

Set $\mathbf{1}=1 \otimes 1 \in V_{\hat{\mathfrak{g}}}(\ell, 0)$ and identify $\mathfrak{g}$ as a subspace of $V_{\hat{\mathfrak{g}}}(\ell, 0)$ through linear map

$$
\mathfrak{g} \ni a \mapsto a(-1) \mathbf{1} \in V_{\hat{\mathfrak{g}}}(\ell, 0) .
$$


Then there exists a vertex algebra structure on $V_{\hat{\mathfrak{g}}}(\ell, 0)$, which is uniquely determined by the condition that $\mathbf{1}$ is the vacuum vector and $Y(a, x)=a(x)$ for $a \in \mathfrak{g}$. Furthermore, $V_{\hat{\mathfrak{g}}}(\ell, 0)$ is a $\mathbb{Z}$-graded vertex algebra such that

$$
V_{\hat{\mathfrak{g}}}(\ell, 0)_{(n)}=0 \text { for } n<0, \quad V_{\hat{\mathfrak{g}}}(\ell, 0)_{(0)}=\mathbb{C} \mathbf{1}, \quad \text { and } V_{\hat{\mathfrak{g}}}(\ell, 0)_{(1)}=\mathfrak{g} .
$$

Let $\Gamma$ be an automorphism group of $(\mathfrak{g},\langle\cdot, \cdot\rangle)$. A simple fact is that every automorphism of $\mathfrak{g}$, which reserves $\langle\cdot, \cdot\rangle$, extends uniquely to an automorphism of vertex algebra $V_{\hat{\mathfrak{g}}}(\ell, 0)$. In view of this, we can and we should view $\Gamma$ as an automorphism group of $V_{\hat{\mathfrak{g}}}(\ell, 0)$ as a $\mathbb{Z}$-graded vertex algebra. From Remark 3.5, for any given linear character $\phi$ of $\Gamma, V_{\hat{\mathfrak{g}}}(\ell, 0)$ has a canonical vertex $\Gamma$-algebra structure.

Recall from Section 2 the affine Lie algebra $\widehat{\mathcal{A}}$, where $\mathcal{A}$ is a subalgebra of $\mathfrak{g l}_{\infty}$ with a basis $\left\{E_{m, n} \mid m+n \in 2 \mathbb{Z}\right\}$. We also recall that

$$
G_{\alpha, m}=E_{m+\alpha, m-\alpha} \quad \text { for } \alpha, m \in \mathbb{Z} .
$$

For any complex number $\ell$, we have a $\mathbb{Z}$-graded vertex algebra $V_{\widehat{\mathcal{A}}}(\ell, 0)$ whose degree-1 subspace is canonically identified with $\mathcal{A}$. In particular, we have

$$
G_{\alpha, m} \in V_{\widehat{\mathcal{A}}}(\ell, 0)_{(1)} \quad \text { for } \alpha, m \in \mathbb{Z} .
$$

Every automorphism of $\mathcal{A}$ viewed as a Lie algebra, which preserves $\langle\cdot, \cdot\rangle$, extends canonically to an automorphism of $\widehat{\mathcal{A}}$ and furthermore to an automorphism of vertex algebra $V_{\widehat{\mathcal{A}}}(\ell, 0)$, preserving the $\mathbb{Z}$-grading. In view of this, the automorphisms $\sigma_{n}$ of $\mathcal{A}$, which was defined in Definition 2.4 and $\tau$ extend to automorphisms of $V_{\widehat{\mathcal{A}}}(\ell, 0)$. Then we view $\mathbb{Z}_{2} \times \mathbb{Z}$ as an automorphism group of $V_{\widehat{\mathcal{A}}}(\ell, 0)$, preserving the $\mathbb{Z}$-grading. For $n \in \mathbb{Z}$, set

$$
R_{n}=q^{-n L(0)} \sigma_{n} \in \operatorname{GL}\left(V_{\widehat{\mathcal{A}}}(\ell, 0)\right),
$$

recalling that $L(0)$ denotes the degree operator of $V_{\widehat{\mathcal{A}}}(\ell, 0)$. Equipped with these structures, $V_{\widehat{\mathcal{A}}}(\ell, 0)$ becomes a vertex $\mathbb{Z}$-algebra.

We formulate the following routine notions:

Definition 3.9. An $\widehat{A}_{\hbar}$-module $W$ is said to be of level $\ell \in \mathbb{C}$ if $c$ acts on $W$ as scalar $\ell$, and it is said to be restricted if for any $w \in W, \alpha \in \mathbb{Z}, A_{\alpha, n} w=0$ for $n$ sufficiently large.

As the main result of this section we have:

Theorem 3.10. Assume that $q$ is not a root of unity and let $\ell \in \mathbb{C}$. Then for any restricted $\widehat{A}_{\hbar}$-module $W$ of level $\ell$, there exists an equivariant quasi $V_{\widehat{\mathcal{A}}}(\ell, 0)$-module structure $Y_{W}(\cdot, x)$ on $W$, which is uniquely determined by

$$
Y_{W}\left(G_{\alpha, m}, x\right)=q^{m} A_{\alpha}\left(q^{m} x\right) \quad \text { for } \alpha, m \in \mathbb{Z} .
$$

On the other hand, for any equivariant quasi $V_{\widehat{\mathcal{A}}}(\ell, 0)$-module $\left(W, Y_{W}\right), W$ becomes a restricted $\widehat{A}_{\hbar}$-module of level $\ell$ with

$$
A_{\alpha}(z)=Y_{W}\left(G_{\alpha, 0}, z\right) \quad \text { for } \alpha \in \mathbb{Z}
$$


Proof. Let $W$ be a restricted $\widehat{A}_{\hbar}$-module of level $\ell$. In view of Proposition 2.7, $W$ is a restricted $\widehat{\mathcal{A}}[\mathbb{Z}]$-module of level $\ell$ with

$$
\overline{G_{\alpha, 0} \otimes t^{m}}=A_{\alpha, m} \quad \text { for } \alpha, m \in \mathbb{Z} .
$$

Note that as $q$ is not a root of unity, $\chi_{q}: \mathbb{Z} \rightarrow \mathbb{C}^{\times}$is one-to-one. By Theorem 4.9 of [Li4, there exists an equivariant quasi $V_{\widehat{\mathcal{A}}}(\ell, 0)$-module structure $Y_{W}(\cdot, x)$ on $W$, which is uniquely determined by

$$
Y_{W}\left(G_{\alpha, m}, x\right)=\overline{G_{\alpha, m}(x)} \quad \text { for } \alpha, m \in \mathbb{Z} .
$$

For $\alpha, m \in \mathbb{Z}$, we have

$$
Y_{W}\left(G_{\alpha, m}, x\right)=\overline{G_{\alpha, m}(x)}=\overline{\sigma_{m} G_{\alpha, 0}(x)}=q^{m} \overline{G_{\alpha, 0}\left(q^{m} x\right)}=q^{m} A_{\alpha}\left(q^{m} x\right) .
$$

Consequently, there exists an equivariant quasi $V_{\widehat{\mathcal{A}}}(\ell, 0)$-module structure $Y_{W}(\cdot, x)$ on $W$ such that

$$
Y_{W}\left(G_{\alpha, m}, x\right)=q^{m} A_{\alpha}\left(q^{m} x\right) \quad \text { for } \alpha, m \in \mathbb{Z} .
$$

The other direction follows from Proposition 2.7 and Theorem 4.9 of [Li4].

Recall the linear character $\chi_{q}^{B}: \mathbb{Z}_{2} \times \mathbb{Z} \rightarrow \mathbb{C}^{\times}$which was defined in Section 2 by

$$
\chi_{q}^{B}(\tau)=-1 \quad \text { and } \quad \chi_{q}^{B}\left(\sigma_{n}\right)=q^{n} \quad \text { for } n \in \mathbb{Z} .
$$

Note that if $q$ is not a root of unity, then $\chi_{q}$ is one-to-one. View $V_{\widehat{A}}(2 \ell, 0)$ as a vertex $\left(\mathbb{Z}_{2} \times \mathbb{Z}\right)$-algebra. Combining Proposition 2.10] with Theorem 4.9 of [Li4] we immediately have:

Theorem 3.11. Assume that $q$ is not a root of unity and let $\ell \in \mathbb{C}$. Then for any restricted $\widehat{B}_{\hbar}$-module $W$ of level $\ell$, there exists a $\left(\mathbb{Z}_{2} \times \mathbb{Z}, \chi_{q}^{B}\right)$-equivariant quasi $V_{\widehat{\mathcal{A}}}(2 \ell, 0)$ module structure $Y_{W}(\cdot, x)$ on $W$, which is uniquely determined by

$$
Y_{W}\left(G_{\alpha, m}, x\right)=q^{m} B_{\alpha}\left(q^{m} x\right) \quad \text { for } \alpha, m \in \mathbb{Z} .
$$

On the other hand, every $\left(\mathbb{Z}_{2} \times \mathbb{Z}, \chi_{q}^{B}\right)$-equivariant quasi $V_{\widehat{\mathcal{A}}}(2 \ell, 0)$-module $W$ is a restricted $\widehat{B}_{\hbar}$-module of level $\ell$ with

$$
B_{\alpha}(z)=Y_{W}\left(G_{\alpha, 0}, z\right) \quad \text { for } \alpha \in \mathbb{Z}
$$

Recall that $\tau\left(E_{m, n}\right)=-E_{n, m}$ for $m, n \in \mathbb{Z}$, where $\tau$ is an order 2 automorphism of the Lie algebra $\mathfrak{g l}_{\infty}$, which preserves the bilinear form. Furthermore, $\tau$ preserves $\mathcal{A}$; $\tau\left(G_{\alpha, m}\right)=-G_{-\alpha, m}$ for $\alpha, m \in \mathbb{Z}$. Recall that $\mathcal{A}^{\tau}$ denotes the Lie subalgebra of $\tau$-fixed points in $\mathcal{A}$. Fix a linear character $\chi_{q}^{D}: \mathbb{Z} \rightarrow \mathbb{C}^{\times}$defined by

$$
\chi_{q}^{D}\left(\sigma_{n}\right)=q^{n} \quad \text { for } n \in \mathbb{Z} .
$$

We have: 
Theorem 3.12. Assume that $q$ is not a root of unity and let $\ell \in \mathbb{C}$. Then for any restricted $\widehat{D}_{\hbar}$-module $W$ of level $\ell$, there exists a $\left(\mathbb{Z}, \chi_{q}^{D}\right)$-equivariant quasi $V_{\widehat{\mathcal{A}}^{\tau}}(\ell, 0)$-module structure $Y_{W}(\cdot, x)$ on $W$, which is uniquely determined by

$$
Y_{W}\left(G_{\alpha, m}^{\tau}, x\right)=q^{-\alpha+m} D_{\alpha}\left(q^{m} x\right) \quad \text { for } \alpha, m \in \mathbb{Z},
$$

where $G_{\alpha, m}^{\tau}=G_{\alpha, m}-G_{-\alpha, m} \in \mathcal{A}^{\tau}$. On the other hand, given a $\left(\mathbb{Z}, \chi_{q}^{D}\right)$-equivariant quasi $V_{\widehat{\mathcal{A}}^{\tau}}(\ell, 0)$-module $\left(W, Y_{W}\right)$, one has a restricted $\widehat{D}_{\hbar}$-module structure of level $\ell$ on $W$ such that $D_{\alpha}(x)=q^{\alpha} Y_{W}\left(G_{\alpha, 0}^{\tau}, x\right)$ for $\alpha \in \mathbb{Z}$.

\section{Vertex algebra $L_{\widehat{\mathcal{A}}}(\ell, 0)$ and its modules}

In this section, we study the vertex algebra $L_{\widehat{\mathcal{A}}}(\ell, 0)$ with $\ell$ a positive integer and give a characterization of $L_{\widehat{\mathcal{A}}}(\ell, 0)$-modules.

We start with the $\mathbb{Z}$-graded vertex algebra $V_{\hat{\mathfrak{g}}}(\ell, 0)$ associated to an affine Lie algebra $\hat{\mathfrak{g}}$ of a (possibly infinite dimensional) Lie algebra $\mathfrak{g}$, where

$$
V_{\hat{\mathfrak{g}}}(\ell, 0)_{(n)}=0 \text { for } n<0, \quad V_{\hat{\mathfrak{g}}}(\ell, 0)_{(0)}=\mathbb{C} \mathbf{1}, \quad \text { and } \quad V_{\hat{\mathfrak{g}}}(\ell, 0)_{(1)}=\mathfrak{g} .
$$

Define a linear operator $\mathcal{D}$ on $V_{\hat{\mathfrak{g}}}(\ell, 0)$ by $\mathcal{D} v=v_{-2} \mathbf{1}$ for $v \in V_{\hat{\mathfrak{g}}}(\ell, 0)$. We have

$$
\mathcal{D} V_{\hat{\mathfrak{g}}}(\ell, 0)_{(n)} \subset V_{\hat{\mathfrak{g}}}(\ell, 0)_{(n+1)} \quad \text { for } n \in \mathbb{Z} .
$$

Lemma 4.1. Let $J_{\hat{\mathfrak{g}}}(\ell, 0)$ be the (unique) maximal graded $\hat{\mathfrak{g}}$-submodule of $V_{\hat{\mathfrak{g}}}(\ell, 0)$. Then $J_{\hat{\mathfrak{g}}}(\ell, 0)$ is a two-sided ideal of $V_{\hat{\mathfrak{g}}}(\ell, 0)$.

Proof. Since $\mathfrak{g}$ generates $V_{\hat{\mathfrak{g}}}(\ell, 0)$ as a vertex algebra, it follows that $J_{\hat{\mathfrak{g}}}(\ell, 0)$ is a left ideal. To prove $J_{\hat{\mathfrak{g}}}(\ell, 0)$ is also a right ideal, from [LL] we must prove $\mathcal{D} J_{\hat{\mathfrak{g}}}(\ell, 0) \subset J_{\hat{\mathfrak{g}}}(\ell, 0)$. Since $\left[\mathcal{D}, a_{n}\right]=-n a_{n-1}$ for $a \in \mathfrak{g}, n \in \mathbb{Z}$, it can be readily seen that $J_{\hat{\mathfrak{g}}}(\ell, 0)+\mathcal{D}\left(J_{\hat{\mathfrak{g}}}(\ell, 0)\right)$ is a graded $\hat{\mathfrak{g}}$-submodule of $V_{\hat{\mathfrak{g}}}(\ell, 0)$. From (4.1) the degree-zero homogeneous subspace of $J_{\hat{\mathfrak{g}}}(\ell, 0)+\mathcal{D}\left(J_{\hat{\mathfrak{g}}}(\ell, 0)\right)$ is trivial. Thus we have $J_{\hat{\mathfrak{g}}}(\ell, 0)+\mathcal{D} J_{\hat{\mathfrak{g}}}(\ell, 0) \subset J_{\hat{\mathfrak{g}}}(\ell, 0)$, proving $\mathcal{D} J_{\hat{\mathfrak{g}}}(\ell, 0) \subset J_{\hat{\mathfrak{g}}}(\ell, 0)$. Therefore, $J_{\hat{\mathfrak{g}}}(\ell, 0)$ is an ideal of $V_{\hat{\mathfrak{g}}}(\ell, 0)$.

Definition 4.2. Denote by $L_{\hat{\mathfrak{g}}}(\ell, 0)$ the quotient vertex algebra of $V_{\hat{\mathfrak{g}}}(\ell, 0)$ modulo $J_{\hat{\mathfrak{g}}}(\ell, 0)$, which is a graded simple vertex algebra.

Let $U$ be a $\mathfrak{g}$-module. We make $U$ a $(\mathfrak{g} \otimes \mathbb{C}[t]+\mathbb{C k})$-module by letting $\mathfrak{g} \otimes t \mathbb{C}[t]$ act trivially and $\mathbf{k}$ act as scalar $\ell$. Then form a generalized Verma $\hat{\mathfrak{g}}$-module

$$
M_{\hat{\mathfrak{g}}}(\ell, U)=U(\hat{\mathfrak{g}}) \otimes_{U(\mathfrak{g} \otimes \mathbb{C}[t]+\mathbb{C k})} U,
$$

which is naturally $\mathbb{N}$-graded. Define $L_{\hat{\mathfrak{g}}}(\ell, U)$ to be the quotient module of $M_{\hat{\mathfrak{g}}}(\ell, U)$ modulo the maximal graded submodule with trivial degree-zero subspace.

Now, specifying $\mathfrak{g}$ to $\mathcal{A}$, where

$$
\mathcal{A}=\operatorname{span}\left\{E_{m, n} \mid m+n \in 2 \mathbb{Z}\right\} \subset \mathfrak{g l}_{\infty},
$$

we have $\mathbb{Z}$-graded vertex algebras $V_{\widehat{\mathcal{A}}}(\ell, 0)$ and $L_{\widehat{\mathcal{A}}}(\ell, 0)$. If $\ell=0$, we see that $\mathcal{A}$ generates a proper graded ideal of $V_{\widehat{\mathcal{A}}}(0,0)$. Consequently, $L_{\widehat{\mathcal{A}}}(0,0)=\mathbb{C}$. If $\ell \neq 0$, from [LL] we have $L_{\widehat{\mathcal{A}}}(\ell, 0)_{(1)}=\mathcal{A}$. 
Remark 4.3. It is straightforward to show that $J_{\widehat{\mathcal{A}}}(\ell, 0)$ is stable under every automorphism of $V_{\widehat{\mathcal{A}}}(\ell, 0)$, which preserves the $\mathbb{Z}$-grading. Thus, $J_{\widehat{\mathcal{A}}}(\ell, 0)$ is stable under the action of group $\mathbb{Z}_{2} \times \mathbb{Z}$ as defined in Section 3 . Consequently, $\mathbb{Z}_{2} \times \mathbb{Z}$ acts on $L_{\widehat{\mathcal{A}}}(\ell, 0)$ by automorphisms that preserve the $\mathbb{Z}$-grading.

Notice that

$$
\mathcal{A}=\operatorname{span}\left\{E_{m, n} \mid m, n \in 2 \mathbb{Z}\right\} \oplus \operatorname{span}\left\{E_{p, q} \mid p, q \in 1+2 \mathbb{Z}\right\},
$$

which is isomorphic to $\mathfrak{g l}_{\infty} \oplus \mathfrak{g l}_{\infty}$ as an associative algebra. As a Lie algebra, $\mathcal{A}$ has a Cartan subalgebra

$$
H=\operatorname{span}\left\{E_{m, m} \mid m \in \mathbb{Z}\right\} .
$$

Recall

$$
G_{\alpha, m}=E_{m+\alpha, m-\alpha} \in \mathcal{A} \quad \text { for } \alpha, m \in \mathbb{Z} .
$$

Then $H=\operatorname{span}\left\{G_{0, m} \mid m \in \mathbb{Z}\right\}$ and for any $\alpha, m \in \mathbb{Z}$ with $\alpha \neq 0, G_{\alpha, m}$ is a root vector of $\mathcal{A}$ viewed as a Lie algebra.

First, we have:

Lemma 4.4. For any complex number $\ell, L_{\widehat{\mathcal{A}}}(\ell, 0)$ is an irreducible $\widehat{\mathcal{A}}$-module and a simple vertex algebra.

Proof. Notice that the second assertion follows from the first one. For the first assertion, we need to show that every $\widehat{\mathcal{A}}$-submodule of $V_{\widehat{\mathcal{A}}}(\ell, 0)$ is graded. If $\ell=0$, we have $L_{\widehat{\mathcal{A}}}(\ell, 0)=$ $\mathbb{C}$, which is irreducible. We now assume $\ell \neq 0$. Let $W$ be an $\widehat{\mathcal{A}}$-submodule of $V_{\widehat{\mathcal{A}}}(\ell, 0)$ and let $v \in W$. Then there exists a finite interval $I$ of $\mathbb{Z}$ such that $v \in U\left(\widehat{\mathcal{A}_{I}}\right) \mathbf{1}$. where $\mathcal{A}_{I}=\operatorname{span}\left\{E_{m, n} \mid m, n \in I, m+n \in 2 \mathbb{Z}\right\}$. We assume that $|I|$ is even. Then $\mathcal{A}_{I}$ is a Lie subalgebra isomorphic to the direct sum of $\mathfrak{s l}_{\frac{1}{2}|I|} \oplus \mathfrak{s l}_{\frac{1}{2}|I|}$ and a two-dimensional abelian algebra. We choose $I$ such that $\ell \neq-\frac{1}{2}|I|$. It is clear that $U\left(\widehat{\mathcal{A}_{I}}\right) \mathbf{1}$ is a graded vertex subalgebra of $V_{\widehat{\mathcal{A}}}(\ell, 0)$, generated by $\mathcal{A}_{I}$. In view of the $\mathrm{P}-\mathrm{B}-\mathrm{W}$ theorem, we have $U\left(\widehat{\mathcal{A}_{I}}\right) \mathbf{1} \simeq V_{\widehat{\mathcal{A}_{I}}}(\ell, 0)$ as a $\mathbb{Z}$-graded $\widehat{\mathcal{A}_{I}}$-module. As $\ell \neq 0$ and $\ell \neq-\frac{1}{2}|I|$ (the negative dual Coxeter number of $\left.\mathfrak{s l}_{\frac{1}{2}|I|}\right), V_{\widehat{\mathcal{A}_{I}}}(\ell, 0)$ is a vertex operator algebra with the standard Segal-Sugawara conformal vector $\omega_{I}$. Furthermore, the $\mathbb{Z}$-grading of $V_{\widehat{\mathcal{A}_{I}}}(\ell, 0)$ by the $L_{I}(0)$ weight coincides with the $\mathbb{Z}$-grading of $V_{\widehat{\mathcal{A}}}(\ell, 0)$. As an $\widehat{\mathcal{A}_{I}}$-submodule, $W$ is stable under the action of $L_{I}(0)$. It follows that every homogeneous component of $v$ lies in $W$. This proves that $W$ is a graded subspace. Therefore, $L_{\widehat{\mathcal{A}}}(\ell, 0)$ is an irreducible $\widehat{\mathcal{A}}$-module.

Definition 4.5. An $\widehat{\mathcal{A}}$-module $W$ is said to be integrable if $H$ is semisimple on $W$ and if for any $\alpha, m, k \in \mathbb{Z}$ with $\alpha \neq 0, G_{\alpha, m}(k)$ is locally nilpotent on $W$.

Lemma 4.6. Let $\ell$ be a complex number and let $W$ be a nonzero restricted $\widehat{\mathcal{A}}$-module of level $\ell$, on which $H$ is semisimple. Then $W$ is integrable if and only if $\ell$ is a nonnegative integer and for any $\alpha, m \in \mathbb{Z}$ with $\alpha \neq 0$,

$$
G_{\alpha, m}(x)^{\ell+1}=0 \quad \text { on } W .
$$


Proof. Assume that $W$ is integrable. Let $m, n \in \mathbb{Z}$ with $m+n \in 2 \mathbb{Z}, m \neq n$. We have a Lie algebra embedding of $\widehat{\mathfrak{s}(2, \mathbb{C})}$ into $\widehat{\mathcal{A}}$ :

$$
\mathbf{k} \mapsto \mathbf{k}, \quad e \otimes t^{r} \mapsto E_{m, n} \otimes t^{r}, \quad f \otimes t^{r} \mapsto E_{n, m} \otimes t^{r}, \quad h \otimes t^{r} \mapsto\left(E_{m, m}-E_{n, n}\right) \otimes t^{r}
$$

for $r \in \mathbb{Z}$. Then $W$ is necessarily a restricted and integrable $\mathfrak{s l ( 2 , \mathbb { C } )}$-module of level $\ell$ which must be a nonnegative integer. From [DLM], $W$ is a direct sum of irreducible integrable highest weight $\widehat{\mathfrak{s l}(2, \mathbb{C})}$-modules. It then follows from $[\mathrm{LP}]$ that

$$
E_{m, n}(x)^{\ell+1}=E_{n, m}(x)^{\ell+1}=0 \quad \text { on } W .
$$

The other direction essentially follows from [DLM]: Assume that $G_{\alpha, m}(x)^{\ell+1}=0$ on $W$ for $\alpha, m \in \mathbb{Z}$ with $\alpha \neq 0$. Let $w \in W$. We need to show that for $n \in \mathbb{Z}, G_{\alpha, m}(n)^{q} w=0$ for some positive integer $q$. This will follow from deduction on $n$ as follows. First, there exists an integer $k$ such that $G_{\alpha, m}(p) w=0$ for $p>k$. Let $A$ be the commutative subalgebra of $U(\widehat{\mathcal{A}})$, generated by $G_{\alpha, m}(n)$ for $n \in \mathbb{Z}$, and set $E=A w \subset W$. We see that $G_{\alpha, m}(n)=0$ on $E$ for $n>k$. Assume that $G_{\alpha, m}(n)$ for $n>k-r$ are all nilpotent on $E$ for some nonnegative integer $r$. By extracting the coefficient of $x^{-(\ell+1)(k-r+1)}$ from $G_{\alpha, m}(x)^{\ell+1}=0$ on $E$, we get

$$
G_{\alpha, m}(k-r)^{\ell+1}=G_{\alpha, m}(k-r) X \quad \text { on } E,
$$

where $X$ is some polynomial of $G_{\alpha, m}(k-p)$ with $p<r$. It follows that $G_{\alpha, m}(k-r)$ is also nilpotent on $E$. This proves that $W$ is an integrable $\widehat{\mathcal{A}}$-module.

For the vertex algebras $V_{\widehat{\mathcal{A}}}(\ell, 0)$ and $L_{\widehat{\mathcal{A}}}(\ell, 0)$, we have:

Proposition 4.7. Let $\ell$ be a nonnegative integer. Then the maximal graded $\widehat{\mathcal{A}}$-submodule $J_{\widehat{\mathcal{A}}}(\ell, 0)$ of $V_{\widehat{\mathcal{A}}}(\ell, 0)$ is generated by $G_{\alpha, m}(-1)^{\ell+1} \mathbf{1}$ for $\alpha, m \in \mathbb{Z}$ with $\alpha \neq 0$. Furthermore, $L_{\widehat{\mathcal{A}}}(\ell, 0)$ is an integrable $\widehat{\mathcal{A}}$-module.

Proof. Let $m, n \in \mathbb{Z}$ with $m+n \in 2 \mathbb{Z}$ and $m \neq n$. Note that $E_{m, n}(-1)^{\ell+1} \mathbf{1}$ is a homogeneous vector of degree $\ell+1$ in $V_{\widehat{\mathcal{A}}}(\ell, 0)$. We claim

$$
E_{p, q}(1) E_{m, n}(-1)^{\ell+1} \mathbf{1}=0 \quad \text { for any } p, q \in \mathbb{Z} \text { with } p+q \in 2 \mathbb{Z}, p \neq q .
$$

If $p \neq n$ and $q \neq m$, we have $\left[E_{p, q}(1), E_{m, n}(-1)\right]=0$, so that

$$
E_{p, q}(1) E_{m, n}(-1)^{\ell+1} \mathbf{1}=E_{m, n}(-1)^{\ell+1} E_{p, q}(1) \mathbf{1}=0 .
$$

Assume $(p, q)=(n, m)$. Notice that $E_{n, m}(1), E_{m, n}(-1), E_{n, n}(0)-E_{m, m}(0)+\ell$ linearly span a Lie subalgebra isomorphic to $\mathfrak{s l}(2, \mathbb{C})$ with

$$
e=E_{m, n}(1), \quad f=E_{n, m}(-1), \quad h=E_{n, n}(0)-E_{m, m}(0)+\ell .
$$

Since

$$
E_{n, m}(1) \mathbf{1}=0, \quad\left(E_{n, n}(0)-E_{m, m}(0)+\ell\right) \mathbf{1}=\ell \mathbf{1},
$$


we have $E_{n, m}(1) E_{m, n}(-1)^{\ell+1} \mathbf{1}=0$.

Consider the case where $q=m$ and $p \neq n$. We have $\left[E_{p, q}(1), E_{m, n}(-1)\right]=E_{p, n}(0)$. As $\left[E_{p, n}(0), E_{m, n}(-1)\right]=0$, we have

$$
E_{p, n}(0) E_{m, n}(-1)^{k} \mathbf{1}=E_{m, n}(-1)^{k} E_{p, n}(0) \mathbf{1}=0
$$

for any nonnegative integer $k$. It follows from induction that

$$
E_{p, q}(1) E_{m, n}(-1)^{k+1} \mathbf{1}=0 \quad \text { for all nonnegative integers } k .
$$

For the case $p=n$ and $q \neq m$, the proof is similar. This proves our claim. It then follows (from the $\mathrm{P}-\mathrm{B}-\mathrm{W}$ theorem) that $U(\widehat{\mathcal{A}}) E_{m, n}(-1)^{\ell+1} \mathbf{1}$ is a proper graded submodule. Therefore

$$
E_{m, n}(-1)^{\ell+1} \mathbf{1} \in J_{\widehat{\mathcal{A}}}(\ell, 0) .
$$

Denote by $J^{\prime}$ the $\widehat{\mathcal{A}}$-submodule of $V_{\widehat{\mathcal{A}}}(\ell, 0)$, generated by $E_{m, n}(-1)^{\ell+1} \mathbf{1}$ for $m, n \in \mathbb{Z}$ with $m+n \in 2 \mathbb{Z}, m \neq n$. Set $V=V_{\widehat{\mathcal{A}}}(\ell, 0) / J^{\prime}$. It follows that for $m, n \in \mathbb{Z}$ with $m+n \in 2 \mathbb{Z}, m \neq n, E_{m, n}(-1)$ is locally nilpotent on $V$. For $k \geq 0$, it is clear that $E_{m, n}(k)$ is locally nilpotent on $V$. Let $I$ be any finite interval of $\mathbb{Z}$ and let $\mathcal{A}_{I}$ be the corresponding subalgebra of $\mathcal{A}$. From $[\mathrm{K}], V$ is an integrable $\widehat{\mathcal{A}_{I}}$-module. As $I$ is arbitrary, it follows that $V$ is an integrable $\widehat{\mathcal{A}}$-module. On the other hand, as a submodule of the integrable $\widehat{\mathcal{A}_{I}}$-module $V, U\left(\widehat{\mathcal{A}_{I}}\right) \mathbf{1}$ must be an irreducible $\widehat{\mathcal{A}_{I}}$-module. It follows that $V$ is an irreducible $\widehat{\mathcal{A}}$-module. Consequently, we have $V=L_{\widehat{\mathcal{A}}}(\ell, 0)$, proving $J^{\prime}=J_{\widehat{\mathcal{A}}}(\ell, 0)$. Then the two assertions hold.

An an immediate consequence of Lemma 4.6 and Proposition 4.7, we have:

Corollary 4.8. Let $\ell$ be a complex number. Then $L_{\widehat{\mathcal{A}}}(\ell, 0)$ is an integrable $\widehat{\mathcal{A}}$-module if and only if $\ell$ is a nonnegative integer.

For the rest of this section, we assume that $\ell$ is a nonnegative integer. Note that each $L_{\widehat{\mathcal{A}}}(\ell, 0)$-module is naturally a restricted $\widehat{\mathcal{A}}$-module of level $\ell$. Furthermore, we have:

Proposition 4.9. All $L_{\widehat{\mathcal{A}}}(\ell, 0)$-modules are exactly those restricted $\widehat{\mathcal{A}}$-modules $W$ of level $\ell$ such that

$$
G_{\alpha, m}(x)^{\ell+1}=0 \quad \text { on } W
$$

for $\alpha, m \in \mathbb{Z}$ with $\alpha \neq 0$.

Proof. Let $\left(W, Y_{W}\right)$ be an $L_{\widehat{\mathcal{A}}}(\ell, 0)$-module. Then $W$ is a restricted $\widehat{\mathcal{A}}$-module of level $\ell$ with $G_{\alpha, m}(x)=Y_{W}\left(G_{\alpha, m}, x\right)$ for $\alpha, m \in \mathbb{Z}$ with $\alpha \neq 0$. From Proposition 4.7 we have $G_{\alpha, m}(-1)^{\ell+1} \mathbf{1}=0$ in $L_{\widehat{\mathcal{A}}}(\ell, 0)$. By a result of Dong-Lepowsky in [DL] we have

$$
G_{\alpha, m}(x)^{\ell+1}=Y_{W}\left(G_{\alpha, m}, x\right)^{\ell+1}=Y_{W}\left(G_{\alpha, m}(-1)^{\ell+1} \mathbf{1}, x\right)=0 \text { on } W .
$$

Conversely, let $W$ be a restricted $\widehat{\mathcal{A}}$-module level $\ell$, satisfying the very property. First, $W$ is naturally a $V_{\widehat{\mathcal{A}}}(\ell, 0)$-module such that $Y_{W}\left(G_{\alpha, m}, x\right)=G_{\alpha, m}(x)$ for $\alpha, m \in \mathbb{Z}$. Furthermore, for $\alpha \neq 0$ we have

$$
Y_{W}\left(G_{\alpha, m}(-1)^{\ell+1} \mathbf{1}, x\right)=Y_{W}\left(G_{\alpha, m}, x\right)^{\ell+1}=G_{\alpha, m}(x)^{\ell+1}=0 .
$$

Then it follows from Proposition 4.7 that $W$ is naturally an $L_{\widehat{\mathcal{A}}}(\ell, 0)$-module. 
As an immediate consequence of Lemma 4.6 and Proposition 4.9, we have:

Corollary 4.10. Let $W$ be a restricted $\widehat{\mathcal{A}}$-module on which $H$ is semisimple. Then $W$ is an $L_{\widehat{\mathcal{A}}}(\ell, 0)$-module if and only if $W$ is an integrable $\widehat{\mathcal{A}}$-module of level $\ell$.

Remark 4.11. Let $\mathfrak{g}$ be a finite-dimensional simple Lie algebra and let $\ell$ be a positive integer. It was known (see [FZ], [DL], [Li1]) that irreducible modules for $L_{\hat{\mathfrak{g}}}(\ell, 0)$ viewed as a vertex operator algebra are exactly integrable highest weight $\hat{\mathfrak{g}}$-modules of level $\ell$. Furthermore, it was proved in [DLM] that every module for $L_{\hat{\mathfrak{g}}}(\ell, 0)$ viewed as a vertex algebra, is a direct sum of integrable highest weight $\hat{\mathfrak{g}}$-modules of level $\ell$.

\section{Quasi modules for simple vertex algebra $L_{\widehat{\mathcal{A}}}(\ell, 0)$}

In this section, we determine irreducible $\Gamma_{q}$-equivariant quasi $L_{\widehat{\mathcal{A}}}(\ell, 0)$-modules and we show that every unitary quasifinite highest weight $\overline{\mathcal{A}}_{\hbar}$-module of level $\ell$ is an irreducible $\Gamma_{q}$-equivariant quasi $L_{\widehat{\mathcal{A}}}(\ell, 0)$-module.

First, we establish a technical result.

Lemma 5.1. Let $\Gamma$ be a group equipped with a linear character $\phi: \Gamma \rightarrow \mathbb{C}^{\times}$. Let $V$ be a vertex $\Gamma$-algebra, let $a, b \in V$ such that $a_{n} b=0$ for $n \geq 0$, and let $\left(W, Y_{W}\right)$ be a $\Gamma$-equivariant quasi $V$-module. Then there exists a polynomial $q(z)$ with $q(1)=1$ such that

$$
q\left(x_{1} / x\right)\left[Y_{W}\left(a, x_{1}\right), Y_{W}(b, x)\right]=0 .
$$

Furthermore, for any such polynomial $q(z)$ we have

$$
Y_{W}\left(a_{-1} b, x\right)=\left.\left(q\left(x_{1} / x\right) Y_{W}\left(a, x_{1}\right) Y_{W}(b, x)\right)\right|_{x_{1}=x} .
$$

Proof. Since $a_{n} b=0$ for $n \geq 0$, from Corollary 3.8 there exist some (possibly the same) nonzero and nonunit complex numbers $\alpha_{1}, \ldots, \alpha_{k}$ such that

$$
\left(x_{1}-\alpha_{1} x_{2}\right) \cdots\left(x_{1}-\alpha_{k} x_{2}\right)\left[Y_{W}\left(a, x_{1}\right), Y_{W}\left(b, x_{2}\right)\right]=0 .
$$

Set $q(z)=\prod_{i=1}^{k}\left(\frac{z-\alpha_{i}}{1-\alpha_{i}}\right)$. Then $q(1)=1$ and (5.1) holds. Note that (5.1) implies

$$
q\left(x_{1} / x\right) Y_{W}\left(a, x_{1}\right) Y_{W}(b, x) \in \operatorname{Hom}\left(W, W\left(\left(x_{1}, x\right)\right)\right) .
$$

In view of Lemma 3.3 , we have

$$
q\left(\left(x+x_{0}\right) / x\right) Y_{W}\left(Y\left(a, x_{0}\right) b, x\right)=\left.\left(q\left(x_{1} / x\right) Y_{W}\left(a, x_{1}\right) Y_{W}(b, x)\right)\right|_{x_{1}=x+x_{0}} .
$$

As $Y\left(a, x_{0}\right) b \in V\left[\left[x_{0}\right]\right]$, we can set $x_{0}=0$, and by doing so we obtain (5.2).

Furthermore, we have: 
Proposition 5.2. Let $V$ be a vertex $\Gamma$-algebra, let $a \in V$ such that $a_{n} a=0$ for $n \geq 0$, and let $\left(W, Y_{W}\right)$ be any $\Gamma$-equivariant quasi $V$-module. Then there exists a polynomial $q(z)$ with $q(1)=1$ such that

$$
q\left(x_{1} / x\right) Y_{W}\left(a, x_{1}\right) Y_{W}(a, x)=q\left(x_{1} / x\right) Y_{W}(a, x) Y_{W}\left(a, x_{1}\right) .
$$

Furthermore, for any such polynomial $q(z)$, we have

$$
\begin{aligned}
& Y_{W}\left(\left(a_{-1}\right)^{\ell+1} \mathbf{1}, x\right) \\
= & \left.\left(P\left(x_{1}, \ldots, x_{\ell}, x\right) Y_{W}\left(a, x_{1}\right) \cdots Y_{W}\left(a, x_{\ell}\right) Y_{W}(a, x)\right)\right|_{x_{1}=x_{2}=\cdots=x_{\ell}=x},
\end{aligned}
$$

where $\ell$ is a positive integer and $P\left(x_{1}, \ldots, x_{\ell+1}\right)=\prod_{1 \leq i<j \leq \ell+1} q\left(x_{i} / x_{j}\right)$.

Proof. Note that the first assertion and the second assertion with $\ell=1$ follow from Lemma 5.1. We now prove the second assertion by induction on $\ell$. For $r \geq 2$, set

$$
P_{r}\left(x_{1}, \ldots, x_{r}\right)=\prod_{1 \leq i<j \leq r} q\left(x_{i} / x_{j}\right)
$$

Then

$$
\begin{aligned}
& P_{r}\left(x_{1}, \ldots, x_{r}\right) Y_{W}\left(a, x_{\sigma(1)}\right) Y_{W}\left(a, x_{\sigma(2)}\right) \cdots Y_{W}\left(a, x_{\sigma(r)}\right) \\
= & P_{r}\left(x_{1}, \ldots, x_{r}\right) Y_{W}\left(a, x_{1}\right) Y_{W}\left(a, x_{2}\right) \cdots Y_{W}\left(a, x_{r}\right)
\end{aligned}
$$

for any permutation $\sigma$ on $\{1,2, \ldots, r\}$. From this we have

$$
P_{r}\left(x_{1}, \ldots, x_{r}\right) Y_{W}\left(a, x_{1}\right) Y_{W}\left(a, x_{2}\right) \cdots Y_{W}\left(a, x_{r}\right) \in \operatorname{Hom}\left(W, W\left(\left(x_{1}, \ldots, x_{r}\right)\right)\right) .
$$

In view of this, the substitution

$$
\left.\left(P_{r}\left(x_{1}, \ldots, x_{r}\right) Y_{W}\left(a, x_{1}\right) Y_{W}\left(a, x_{2}\right) \cdots Y_{W}\left(a, x_{r}\right)\right)\right|_{x_{1}=\cdots=x_{r}=x}
$$

exists in $\operatorname{Hom}(W, W((x)))$.

Now assume $\ell \geq 2$ and set $b=\left(a_{-1}\right)^{\ell} \mathbf{1}$. Note that the assumption $a_{j} a=0$ for $j \geq 0$ is equivalent to that $\left[a_{m}, a_{n}\right]=0$ for $m, n \in \mathbb{Z}$. Then we have $a_{n} b=0$ for all $n \geq 0$ as $\left[a_{n}, a_{-1}\right]=0$ and $a_{n} \mathbf{1}=0$. By induction hypothesis, we have

$$
Y_{W}(b, x)=\left.\left(P_{\ell}\left(x_{2}, \ldots, x_{\ell}, x\right) Y_{W}\left(a, x_{2}\right) \cdots Y_{W}\left(a, x_{\ell}\right) Y_{W}(a, x)\right)\right|_{x_{2}=\cdots=x_{\ell}=x} .
$$

Noticing that

$$
P_{\ell+1}\left(x_{1}, \ldots, x_{\ell}, x\right)=P_{\ell}\left(x_{2}, \ldots, x_{\ell}, x\right) q\left(x_{1} / x\right) \prod_{j=2}^{\ell} q\left(x_{1} / x_{j}\right),
$$

using (5.6) and (15.3) we get

$$
q\left(x_{1} / x\right)^{\ell} Y_{W}\left(a, x_{1}\right) Y_{W}(b, x)=q\left(x_{1} / x\right)^{\ell} Y_{W}(b, x) Y_{W}\left(a, x_{1}\right) .
$$


By Lemma 5.1 we have

$$
Y_{W}\left(a_{-1} b, x\right)=\left.\left(q\left(x_{1} / x\right)^{\ell} Y_{W}\left(a, x_{1}\right) Y_{W}(b, x)\right)\right|_{x_{1}=x} .
$$

Then we obtain

$$
\begin{aligned}
& \left.\left(P_{\ell+1}\left(x_{1}, \ldots, x_{\ell}, x\right) Y_{W}\left(a, x_{1}\right) \cdots Y_{W}\left(a, x_{\ell}\right) Y_{W}(a, x)\right)\right|_{x_{1}=\cdots=x_{\ell}=x} \\
= & \left.\left(q\left(x_{1} / x\right)^{\ell} Y_{W}\left(a, x_{1}\right) P_{\ell}\left(x_{2}, \ldots, x_{\ell}, x\right) Y_{W}\left(a, x_{2}\right) \cdots Y_{W}\left(a, x_{\ell}\right) Y_{W}(a, x)\right)\right|_{x_{1}=\cdots=x_{\ell}=x} \\
= & \left.\left(q\left(x_{1} / x\right)^{\ell} Y_{W}\left(a, x_{1}\right) Y_{W}(b, x)\right)\right|_{x_{1}=x} \\
= & Y_{W}\left(a_{-1} b, x\right) \\
= & Y_{W}\left(\left(a_{-1}\right)^{\ell+1} \mathbf{1}, x\right),
\end{aligned}
$$

as desired.

As an immediate consequence, we have (cf. [DL], [LL], [DM]):

Corollary 5.3. Let $V$ be a vertex $\Gamma$-algebra, let $a \in V$ such that $a_{n} a=0$ for $n \geq 0$, and let $\ell$ be a positive integer. Let $\left(W, Y_{W}\right)$ be a $\Gamma$-equivariant quasi $V$-module and let $q(z)$ be a polynomial as in Proposition 5.2. If $\left(a_{-1}\right)^{\ell+1} \mathbf{1}=0$ in $V$, then

$$
\begin{aligned}
& \left(\prod_{1 \leq i<j \leq \ell+1} q\left(x_{i} / x_{j}\right)\right) Y_{W}\left(a, x_{1}\right) \cdots Y_{W}\left(a, x_{\ell}\right) Y_{W}\left(a, x_{\ell+1}\right) \\
\in & \operatorname{Hom}\left(W, W\left(\left(x_{1}, x_{2}, \ldots, x_{\ell+1}\right)\right)\right)
\end{aligned}
$$

and

$$
\left.\left(\left(\prod_{1 \leq i<j \leq \ell+1} q\left(x_{i} / x_{j}\right)\right) Y_{W}\left(a, x_{1}\right) \cdots Y_{W}\left(a, x_{\ell}\right) Y_{W}\left(a, x_{\ell+1}\right)\right)\right|_{x_{1}=\cdots=x_{\ell}=x_{\ell+1}}=0 .
$$

On the other hand, if (5.8) holds and if $\left(W, Y_{W}\right)$ is faithful, then $\left(a_{-1}\right)^{\ell+1} \mathbf{1}=0$.

Now, we consider the vertex $\Gamma_{q^{-}}$algebra $L_{\widehat{\mathcal{A}}}(\ell, 0)$, recalling that $\Gamma_{q}=\left\{q^{n} \mid n \in \mathbb{Z}\right\}$.

Proposition 5.4. For any restricted $\hat{A}_{\hbar}$-module $W$ and for any $\alpha \in \mathbb{Z}$,

$$
\left(\prod_{1 \leq i<j \leq k+1}\left(x_{i} / x_{j}-q^{2 \alpha}\right)\left(x_{i} / x_{j}-q^{-2 \alpha}\right)\right) A_{\alpha}\left(x_{1}\right) \cdots A_{\alpha}\left(x_{k+1}\right)
$$

lies in $\operatorname{Hom}\left(W, W\left(\left(x_{1}, x_{2}, \ldots, x_{k+1}\right)\right)\right)$ for any positive integer $k$. Furthermore, for a positive integer $\ell, \Gamma_{q}$-equivariant quasi $L_{\widehat{\mathcal{A}}}(\ell, 0)$-modules are exactly those restricted $\hat{A}_{\hbar-}$ modules $W$ of level $\ell$ such that for every nonzero $\alpha \in \mathbb{Z}$,

$$
\left(\prod_{1 \leq i<j \leq \ell+1}\left(x_{i} / x_{j}-q^{2 \alpha}\right)\left(x_{i} / x_{j}-q^{-2 \alpha}\right)\right) A_{\alpha}\left(x_{1}\right) \cdots A_{\alpha}\left(x_{\ell+1}\right)
$$

vanishes at $x_{i} / x_{j}=1$ for $1 \leq i<j \leq \ell+1$. 
Proof. From (2.5) we have

$$
\left(x-q^{\alpha+\beta} z\right)\left(x-q^{-(\alpha+\beta)} z\right)\left[A_{\alpha}(x), A_{\beta}(z)\right]=0
$$

for $\alpha, \beta \in \mathbb{Z}$ (regardless whether $\alpha+\beta=0$ ). In particular, we have

$$
\left(x / z-q^{2 \alpha}\right)\left(x / z-q^{-2 \alpha}\right)\left[A_{\alpha}(x), A_{\alpha}(z)\right]=0 .
$$

Then the first assertion follows. As for the second assertion, in view of Proposition 4.7, $\Gamma_{q^{-}}$ equivariant quasi $L_{\widehat{\mathcal{A}}}(\ell, 0)$-modules are exactly those $\Gamma_{q}$-equivariant quasi $V_{\widehat{\mathcal{A}}}(\ell, 0)$-modules $W$ on which $Y_{W}\left(G_{\alpha, m}(-1)^{\ell+1} \mathbf{1}, x\right)=0$ for all $\alpha, m \in \mathbb{Z}$ with $\alpha \neq 0$. On the other hand, by Theorem 3.10, $\Gamma_{q}$-equivariant quasi $V_{\widehat{\mathcal{A}}}(\ell, 0)$-modules are restricted $\widehat{\mathcal{A}}_{\hbar}$-modules of level $\ell$. For $\alpha, m \in \mathbb{Z}$ with $\alpha \neq 0$, we have

$$
\left(G_{\alpha, m}\right)_{j} G_{\alpha, m}=0 \quad \text { in } V_{\widehat{\mathcal{A}}}(\ell, 0) \quad \text { for } j \geq 0 .
$$

Then the second assertion follows immediately from Corollary [5.3.

Define an anti-involution $\omega$ of $\widehat{A}_{\hbar}$ by

$$
\omega(\mathbf{c})=\mathbf{c}, \quad \omega\left(A_{\alpha, m}\right)=A_{\alpha,-m} \quad \text { for } \alpha, m \in \mathbb{Z} .
$$

From [KR], a quasifinite module $L(\ell, \lambda)$ is unitary if and only if there are finitely many positive integers $n_{i}$ and real numbers $\mu_{i}$ such that $\ell=\sum_{i} n_{i}$ and

$$
\lambda\left(A_{n, 0}\right)=\frac{q^{n}}{q^{n}-q^{-n}} \sum_{i} n_{i} e^{n \mu_{i}}
$$

for every nonzero integer $n$. In particular, unitarity implies that $\ell$ is a nonnegative integer. If $L(\ell, \lambda)$ is a unitary quasifinite module of level 1 , then

$$
\lambda\left(A_{n, 0}\right)=e^{n \mu} \cdot \frac{q^{n}}{q^{n}-q^{-n}}
$$

for $n \in \mathbb{Z} \backslash\{0\}$, where $\mu$ is a real number.

Remark 5.5. Recall from $\left[\mathrm{KR}\right.$ the Lie algebra $\widehat{\mathcal{D}_{q}}$, which has a basis consisting of $C$ (a central element) and $T_{m, n}(m, n \in \mathbb{Z})$, with commutator relations

$$
\left[T_{m, n}, T_{m^{\prime}, n^{\prime}}\right]=2 \sinh \left(h\left(m^{\prime} n-m n^{\prime}\right)\right) T_{m+m^{\prime}, n+n^{\prime}}+m \delta_{m+m^{\prime}, 0} \delta_{n+n^{\prime}, 0} C
$$

for $m, n, m^{\prime}, n^{\prime} \in \mathbb{Z}$, where $q=e^{2 h}$. Lie algebras $\widehat{\mathcal{D}_{q}}$ and $\widehat{A}_{\hbar}$ are isomorphic, where an isomorphism from $\widehat{\mathcal{D}_{q}}$ to $\widehat{A}_{\hbar}$ is given by

$$
C \mapsto \mathbf{c}, \quad T_{m, n} \mapsto-A_{n, m} \quad \text { for } m, n \in \mathbb{Z},
$$


and by identifying $e^{h}$ with $e^{i \hbar}$. For a weight $\lambda \in H^{*}$, set

$$
\Delta_{\lambda}(x)=\sum_{n \neq 0} \Delta_{n}(\lambda) x^{-n}
$$

where for $n \neq 0$,

$$
\Delta_{n}(\lambda)=\lambda\left(T_{0, n}\right)=-\lambda\left(A_{n, 0}\right) .
$$

It was proved in $[\mathrm{KR}]$ that an irreducible highest weight $\widehat{\mathcal{D}_{q}}$-module $L(c, \lambda)$ is quasifinite if and only if there exists a nonzero polynomial $b(x)$ such that

$$
b(x)\left(\Delta_{\lambda}(x)-\Delta_{\lambda}\left(q^{-1} x\right)+c\right)=0 .
$$

Furthermore, it was proved that $L(c, \lambda)$ is unitary if and only if there exist finitely many positive integers $n_{i}$ and real numbers $a_{i}$ such that $c=\sum_{i} n_{i}$ and

$$
\lambda\left(T_{0, n}\right)=\sum_{i} \frac{n_{i} q^{n a_{i}}}{1-q^{n}} \quad \text { for all } n \in \mathbb{Z} \backslash\{0\} .
$$

Correspondingly, for $\widehat{A}_{\hbar}$ we have

$$
\lambda\left(A_{n, 0}\right)=-\sum_{i} \frac{n_{i} q^{2 n a_{i}}}{1-q^{2 n}}=\sum_{i} n_{i} q^{2 n\left(a_{i}-1\right)} \frac{q^{n}}{q^{n}-q^{-n}}
$$

(noticing that $q=e^{2 h}$ for $\widehat{\mathcal{D}_{q}}$ whereas $q=e^{i \hbar}$ for $\widehat{A}_{\hbar}$ ).

As the main result of this section we have:

Theorem 5.6. Let $\ell$ be a positive integer. Then every unitary quasifinite highest weight irreducible $\widehat{A}_{\hbar}$-module of level $\ell$ is an irreducible $\Gamma_{q}$-equivariant quasi $L_{\widehat{\mathcal{A}}}(\ell, 0)$-module.

Proof. We here shall apply Proposition 5.4. First we consider the case $\ell=1$. Set

$$
M(1)=\mathbb{C}\left[x_{1}, x_{2}, x_{3}, \ldots\right],
$$

the algebra of polynomials in variables $x_{1}, x_{2}, \ldots$ Let $\mu \in \mathbb{R}$. For $\alpha \in \mathbb{Z} \backslash\{0\}$, set

$$
\widehat{X}_{\alpha}(z)=a_{\alpha} \exp \left(\sum_{m \geq 1} z^{m}\left(q^{m \alpha}-q^{-m \alpha}\right) x_{m}\right) \exp \left(\sum_{m \geq 1} \frac{z^{-m}}{m}\left(q^{m \alpha}-q^{-m \alpha}\right) \frac{\partial}{\partial x_{m}}\right)
$$

where $q=e^{i \hbar}$ as before and $a_{\alpha}=e^{i \mu \alpha} \frac{q^{\alpha}}{q^{\alpha}-q^{-\alpha}} \in \mathbb{C}$. It was proved in G-KL2] that $M(1)$ becomes an irreducible highest weight $\widehat{A}_{\hbar}$-module of level 1 with

$$
\begin{aligned}
& A_{0, m}=\frac{\partial}{\partial x_{m}}, \quad A_{0,-m}=m x_{m} \quad \text { for } m \geq 1, \\
& A_{\alpha}(z)=\widehat{X}_{\alpha}(z) \quad \text { for } \alpha \in \mathbb{Z} \backslash\{0\},
\end{aligned}
$$


where the highest weight $\lambda$ is determined by $\lambda_{0}=0$ and $\lambda_{\alpha}=a_{\alpha}$ for $\alpha \in \mathbb{Z} \backslash\{0\}$. Denote this $\widehat{A}_{\hbar}$-module by $M(1)^{[\mu]}$. From [G-KL2], for $\alpha, \beta \in \mathbb{Z} \backslash\{0\}$ we have

$$
\widehat{X}_{\alpha}(z) \widehat{X}_{\beta}(w)=\frac{\left(z-q^{\alpha-\beta} w\right)\left(z-q^{\beta-\alpha} w\right)}{\left(z-q^{\alpha+\beta} w\right)\left(z-q^{-(\alpha+\beta)} w\right)} \circ \widehat{X}_{\alpha}(z) \widehat{X}_{\beta}(w) \stackrel{\circ}{\circ} .
$$

That is,

$$
\begin{aligned}
& \left(\frac{z}{w}-q^{\alpha+\beta}\right)\left(\frac{z}{w}-q^{-(\alpha+\beta)}\right) \widehat{X}_{\alpha}(z) \widehat{X}_{\beta}(w) \\
= & \left(\frac{z}{w}-q^{\alpha-\beta}\right)\left(\frac{z}{w}-q^{\beta-\alpha}\right){ }_{\circ}^{\circ} \widehat{X}_{\alpha}(z) \widehat{X}_{\beta}(w)_{\circ}^{\circ} .
\end{aligned}
$$

From this we have

$$
\left(1-\frac{q^{2 \alpha} w}{z}\right)\left(1-\frac{q^{-2 \alpha} w}{z}\right) \widehat{X}_{\alpha}(z) \widehat{X}_{\alpha}(w) \in \operatorname{Hom}(W, W((z, w)))
$$

and

$$
\left.\left(\left(1-\frac{q^{2 \alpha} w}{z}\right)\left(1-\frac{q^{-2 \alpha} w}{z}\right) \widehat{X}_{\alpha}(z) \widehat{X}_{\alpha}(w)\right)\right|_{z=w}=0 .
$$

Furthermore, for $m \in \mathbb{Z}$, we have

$$
\left(1-q^{2 \alpha} \frac{w}{z}\right)\left(1-q^{-2 \alpha} \frac{w}{z}\right) \widehat{X}_{\alpha}\left(q^{m} z\right) \widehat{X}_{\alpha}\left(q^{m} w\right) \in \operatorname{Hom}(W, W((z, w)))
$$

and it vanishes at $z=w$. It follows from Proposition 5.4 that $M(1)^{[\mu]}$ is an irreducible $\Gamma_{q}$-equivariant quasi $L_{\widehat{\mathcal{A}}}(1,0)$-module. On the other hand, it was proved in $[\mathrm{KR}]$ that every unitary quasifinite highest weight irreducible $\widehat{A}_{\hbar}$-module of level 1 is of this form. This proves the theorem for $\ell=1$.

As for the high level case (with $\ell>1$ ), we apply the common trick-to use tensor products. Let $L(\ell, \lambda)$ be a unitary quasifinite irreducible highest weight $\widehat{A}_{\hbar}$-module of level $\ell$. Then from Remark 5.5 there exist finitely many positive integers $n_{j}$ and real numbers $\mu_{j}$ such that $\ell=\sum_{j} n_{j}$ and

$$
\lambda\left(A_{n, 0}\right)=\frac{q^{n}}{q^{n}-q^{-n}} \sum_{j} n_{j} e^{i n \mu_{j}}
$$

for all $n \in \mathbb{Z} \backslash\{0\}$. Consider the tensor product $\widehat{A}_{\hbar}$-module

$$
M=\otimes_{j}\left(M(1)^{\left[\mu_{j}\right]}\right)^{\otimes n_{j}},
$$

which is a restricted $\widehat{A}_{\hbar}$-module of level $\ell$. For every nonzero $\alpha \in \mathbb{Z}$,

$$
\left(\prod_{1 \leq i<j \leq \ell+1}\left(x_{i} / x_{j}-q^{2 \alpha}\right)\left(x_{i} / x_{j}-q^{-2 \alpha}\right)\right) A_{\alpha}\left(x_{1}\right) \cdots A_{\alpha}\left(x_{\ell+1}\right)
$$


vanishes on $M$ at $x_{i} / x_{j}=1$ for $1 \leq i<j \leq \ell+1$. Then it follows from Proposition 5.4 that $M$ is a $\Gamma_{q}$-equivariant quasi $L_{\widehat{\mathcal{A}}}(\ell, 0)$-module. Set $v=\otimes_{j} 1^{\otimes n_{j}} \in M$. We see that $v$ is a highest weight vector of weight $\lambda$. It follows from unitarity that the submodule $U\left(\widehat{A}_{\hbar}\right) v$ generated by $v$ is an irreducible module. Consequently, $U\left(\widehat{A}_{\hbar}\right) v$ is isomorphic to $L(\ell, \lambda)$. Thus $L(\ell, \lambda)$ is a $\Gamma_{q}$-equivariant quasi $L_{\widehat{\mathcal{A}}}(\ell, 0)$-module.

We end up this paper with the following:

Conjecture 5.7. Every $\mathbb{N}$-graded irreducible $\Gamma_{q}$-equivariant quasi $L_{\widehat{\mathcal{A}}}(\ell, 0)$-module is a unitary quasifinite highest weight irreducible $\widehat{A}_{\hbar}$-module of level $\ell$.

\section{References}

[DL] C. Dong and J. Lepowsky, Generalized Vertex Algebras and Relative Vertex Operators, Progress in Math., Vol. 112, Birkhäuser, Boston, 1993.

[DM] J. Ding and Miwa, Zeros and poles of quantum current operators and the condition of quantum integrability, arXiv:q-alg/9608001.

[DF] J. Ding and B. Feigin, Quantum current operators (III): Commutative quantum current operators, semi-infinite construction and functional models, arXiv: q-alg/9612009

[DLM] C. Dong, H.-S. Li and G. Mason, Regularity of rational vertex operator algebras, Adv. Math. 132 (1997) 148-166

[FFZ] D. Fairlie, P. Fletcher, C. Zachos, Trigonometric structure constants for new infinite-dimensional algebras, Phys. Lett. B 218 (1989) 203-206.

[F] E. G. Floratos, Spin wedge and vertex operator representations of trigonometric algebras and their central extensions, Phys. Lett. B 232 (1989) 467-474.

[FLM] I. B. Frenkel, J. Lepowsky and A. Meurman, Vertex Operator Algebras and the Monster, Pure and Applied Math., Vol. 134, Academic Press, Boston, 1988.

[FZ] I. B. Frenkel and Y.-C. Zhu, Vertex operator algebras associated to representations of affine and Virasoro algebras, Duke Math. J. 66 (1992) 123-168.

[G-KK] M. Golenishcheva-Kutuzova and V. Kac, Г-conformal algebras, J. Math. Phys. 39 (1998) 2290-2305.

[G-KL1] M. Golenishcheva-Kutuzova and D. Lebedev, $\mathbb{Z}$-graded trigonometric Lie subalgebras in $\hat{A}_{\infty}, \hat{B}_{\infty}, \hat{C}_{\infty}$, and $\hat{D}_{\infty}$ and their vertex operator representations, Funktsional'nyi Analiz i Ego Prilozhenniya 27 (1993) 12-24.

[G-KL2] M. Golenishcheva-Kutuzova and D. Lebedev, Vertex operator representation of some quantum tori Lie algebras, Commun. Math. Phys. 148 (1992) 403-416. 
[H] J. Hoppe, Diff $T_{A}^{2}$ and the curvature of some infinite-dimensional manifolds, Phys. Lett. B 215 (1988) 706-710.

[K] V. G. Kac, Infinite-dimensional Lie Algebras, 3rd ed., Cambridge Univ. Press, Cambridge, 1990.

[KR] V. G. Kac and A. Radul, Quasifinite highest weight modules over the Lie algebra of differential operators on the circle, Commun. Math. Phys. 157 (1993) 429-457.

[LL] J. Lepowsky and H.-S. Li, Introduction to Vertex Operator Algebras and Their Representations, Progress in Math. 227, Birkhäuser, Boston, 2004.

[LP] J. Lepowsky and M. Primc, Structure of the Standard Modules for the Affine Lie Algebra $A_{1}^{(1)}$, Contemporary Math. 46, Amer. Math. Soc., Providence, 1985.

[Li1] H.-S. Li, Local systems of vertex operators, vertex superalgebras and modules, J. Pure Appl. Algebra 109 (1996) 143-195.

[Li2] H.-S. Li, Local systems of twisted vertex operators, vertex superalgebras and twisted modules, Contemporary Math. 193, Amer. Math. Soc., Providence, 1996, 203-236.

[Li3] H.-S. Li, A new construction of vertex algebras and quasi modules for vertex algebras, Adv. Math. 202 (2006) 232-286.

[Li4] H.-S. Li, On certain generalizations of twisted affine Lie algebras and quasimodules for Г-vertex algebras, J. Pure Appl. Algebra 209 (2007) 853-871.

[Li5] H.-S. Li, Twisted modules and quasi-modules for vertex operator algebras, Contemporary Math. 422, Amer. Math. Soc., Providence, 2007, 389-400.

[LTW] H.-S. Li, S.-B. Tan and Q. Wang, Twisted modules for quantum vertex algebras, J. Pure Appl. Algebra 214 (2010) 201-220.

[MP1] A. Meurman and M. Primc, Vertex operator algebras and representations of affine Lie algebras, Acta Applicandae Math. 44 (1996), 207-215.

[MP2] A. Meurman and M. Primc, Annihilating Fields of Standard Modules of $\widetilde{\mathfrak{s}(2, \mathbb{C})}$ and Combinatorial Identities, preprint 1994; Memoirs Amer. Math. Soc. 652, 1999. 